\title{
Guidelines for Radiation Safety in Interventional Cardiology (JCS 2006) \\ - Digest Version -
}

JCS Joint Working Group

\section{Table of Contents}

I General Issues ………………................................ 2761

1. In Formulating the Guidelines .............................. 2761

2. Basic Knowledge of Radiation Exposure Control: Stochastic and Deterministic Effects, Absorbed Dose, Effective Dose, and Dose Units (Gy/Sv) .... 2761 A Differences Between Patient Exposure and Exposure of Medical Personnel ............................................. 2761

B Stochastic Effects and Deterministic Effects ...................22761

C Measuring Radiation (Radiometry) ……....................... 2761

(1) Tissue-Absorbed Dose …..................................... 2761

(2) Equivalent Dose ……….................................... 2762

(3) Effective Dose …….......................................... 2762

3. Classification by Severity of Radiation-Induced

Skin Injuries and Clinical Course ………………..... 2762

II Specific Issues (Qs and As) …….................... 2763

1. Basic Knowledge of Radiation-Induced Skin

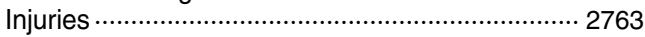

A Radiation-Induced Skin Injuries in $\mathrm{PCl}$......................... 2763

B Areas Where Radiation-Induced Skin Injuries Are Most

Likely to Occur ……............................................... 2764

2. Informed Consent and Countermeasures in Case

of Excess Exposure and Onset of Radiation-

Induced Skin Injuries ……………………………..... 2764

A Matters to Be Included in the Explanation of Radiation

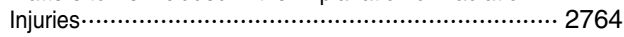

B Explanation and Consent During PCI Procedure

and Subsequent Measures ….................................. 2764

C Explanation of and Measures Taken After Exposure

to Excessive Doses ………………………………... 2765

D Measures Taken in Case of Onset of Radiation-Induced

Skin Injuries ……...................................................... 2766

3. Variables Affecting Exposure Doses...................... 2766

A Effects of Irradiation Pulse Rate …………………..... 2766

B Effects of Patient Body Type …………………….... 2767

C Distance Between Image Intensifier and Patient .............. 2768

D Distance Between X-Ray Tube and Patient ………….... 2768

E Size of Transillumination Field …………………….... 2768

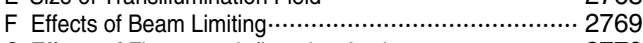

G Effects of Fluoroscopic/lmaging Angle ....................... 2770

H Flat Panel Detector (FPD) Type Imaging System ……... 2770

I Effects of Pacemakers and Leads ………………….... 2771

$\mathrm{J}$ Effects of the Upper Arm in the Irradiation Field

on Fluoroscopy/lmaging …………………………... 2771

4. Efforts Toward Reduction of Patient Exposure

Dose …........................................................... 2773

A General Rules for Reduction of Exposure Dose ………. 2773

B Exposure During Emergency Examination/Treatment …2 2773
5. Patient Variables Affecting the Onset

of Radiation-Induced Skin Injuries ...

6. Strategies for Reducing Exposure in Medical

Personnel ............................................................ 2774

A Medical Personnel Exposure ………………………..... 2774

B How to Use Personal Dosimeters ……....................... 2774

C Types and Effects of Protective Clothing and Effects

of Lead Equivalent ……....................................... 2774

D Maintenance and Management of Protective Clothing … 2776

E Effects of Goggles and Neck Protectors ……………..... 2776

F Protective Devices Recommended for Installation

in the Imaging Laboratory and How to Use Them ……... 2777

G Dose Distribution in the Catheterization Laboratory ……. 2779

H Pregnancy of Female Medical Personnel..................... 2779

I Education and Re-Education of Medical Personnel........ 2779

$\checkmark$ Requirements for Labor Accident Indemnification

Approval …...................................................... 2779

7. Management of Imaging Systems........................ 2780

A Maintenance and Management of Imaging Systems ….... 2780

B Measures to Reduce Exposure ………....................... 2780

8. Non-Coronary Intervention …………………..... 2780

A Precautions in Head and Neck IVR .............................. 2780

B Precautions in Percutaneous Transluminal Angioplasty

(PTA) in Lower Limbs …………………………….... 2780

9. Electrophysiological Examinations and

Treatments …...................................................... 2781

A Exposure Doses to Patients Undergoing Catheter

Ablation …......................................................... 2781

B Precautions for Pediatric Patients ……......................... 2781

10. Nuclear Imaging …………………........................... 2781

A Precautions in Performing $\mathrm{PCl}$ on the Same Day as Thallium (TI) Myocardial Scintigraphy or the Following Day …………………...................... 2781

B Exposure of Medical Personnel Engaged in TI

Myocardial Scintigraphy …………………………..... 2781

C How to Handle Subcutaneous Leakage of Isotopes ….... 2781

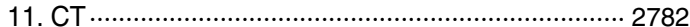

A Exposure Dose to Patients Undergoing Coronary CT …. 2782

B Effects of the Use of Multi-Row Detectors …………….... 2784

C Effects on Pacemaker and Implantable Cardioverter



12. Examination and Treatment of Pregnant

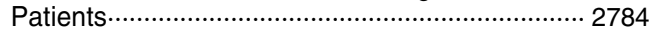

References.............................................................. 2784

(Circ J 2010; 74: 2760-2785)

Released online November 18, 2010

Mailing address: Scientific Committee of the Japanese Circulation Society, 8th Floor CUBE OIKE Bldg., 599 Bano-cho, Karasuma Aneyakoji, Nakagyo-ku, Kyoto 604-8172, Japan. E-mail: meeting@j-circ.or.jp

This English language document is a digest version of Guidelines for Radiation Safety in Interventional Cardiology reported at the Japanese Circulation Society Joint Working Groups performed in 2004-2005 (Circulation Journal 2006; 70(Suppl IV): 1301 - 1327).

Joint Working Groups: The Japanese Circulation Society, The Japanese Coronary Association, The Japanese Society of Interventional Cardiology, The Japanese College of Cardiology, The Japanese Association of Cardiovascular Catheterization Therapeutics, The Japanese Society of Electrocardiology, The Japanese Dermatological Association, The Japanese Heart Rhythm Society, The Japanese Society of Radiation Safety Management, The Japanese Society of Radiological Technology

ISSN-1346-9843 doi:10.1253/circj.CJ-88-0002

All rights are reserved to the Japanese Circulation Society. For permissions, please e-mail: cj@j-circ.or.jp 


\section{General Issues}

\section{In Formulating the Guidelines}

As the prevalence of interventional procedures to treat cardiovascular diseases increases, cases of radiation-induced skin injury among patients who undergo cardiovascular intervention are increasing rapidly. Warnings concerning skin injuries associated with interventional cardiology have been issued by the US Food and Drug Administration (FDA) and the Japan Radiological Society since the mid-1990s. However, cardiovascular physicians have generally not been well aware of radiation-induced skin injuries, possibly because of their interest mainly in life-threatening diseases such as ischemic heart disease and arrhythmias. Interventional cardiology has become increasingly sophisticated, and the indications for it have expanded. This trend has resulted in an increased number of radiation-induced skin injuries and other types of health hazards, such as cataract and hypothyroidism, in those who perform the procedures.

Since not all physicians engaged in interventional cardiology have basic knowledge of radiation, appropriate education and training programs are required. For nurses and medical technologists working in departments other than radiology as well, it is important to have a good understanding of exposure doses that can occur during work hours and their effects. In this situation, "the Guidelines for Prevention of Radiation-induced Skin Injuries Associated with Interventional Radiology (IVR)" were formulated in 2001 by the Japan Association on Radiological Protection in Medicine with the participation of 13 academic institutions. The Guidelines describe relevant issues in a readily understandable fashion, including Q-and-A lessons, and are quite useful for cardiovascular physicians as well. Unfortunately, however, cardiovascular physicians are not in general awareness of their availability. In addition, there has been a demand for more readily understandable guidelines including answers to common questions shared by cardiovascular physicians, procedures characteristics of cardiovascular medicine, such as percutaneous coronary intervention (PCI) and ablation, the best mode of informed consent, and other issues. In the US, similar guidelines for cardiovascular physicians have been formulated and published.

Members of the Japan Association on Radiological Protection in Medicine made significant contributions to the formulation of the present guidelines. Featuring Q-and-A lessons with many straightforward tables and figures, the guidelines are believed to help educate and train cardiovascular physicians who are not specialized in radiology. We hope that the present guidelines will be used in the best way possible for the sake of the safety of patients and medical personnel. ${ }^{1}$

\section{Basic Knowledge of Radiation Exposure Control: Stochastic and Deterministic Effects, Absorbed Dose, Effective Dose, and Dose Units (Gy/Sv)}

\section{A Differences Between Patient Exposure and Exposure of Medical Personnel}

Cardiac catheterization is a procedure performed for diagnostic and therapeutic purposes by means of images obtained by the delivery of $\mathrm{X}$-irradiation to the patient. The X-rays from the X-ray system (X-ray tube focus) delivered directly to the patient during this procedure are termed primary X-rays. The X-rays that have entered the patient's body collide with the orbital electrons of atoms in the body and exhibit interactions, such as flipping of orbital electrons with loss of energy (photoelectric effect) and scattering in directions other than the direction of entrance (Compton scattering). The X-rays emitted from the body as a result of these interactions are termed secondary $\mathbf{X}$-rays.

A fact requiring special attention is that while the $X$-ray tube is the source of radiation causing exposure to the patient, the secondary rays from the patient's body make a much larger contribution to exposure of the operator.

\section{B Stochastic Effects and Deterministic Effects}

Most organs and tissues remain unaffected by the loss of a considerable number of cells. If the number reaches a critical level, however, impairment of tissue function results in an observable disorder. Although the probability of causing such a disorder is nearly zero at low doses of radiation, it rises to $100 \%$ rapidly after a given level of dose (threshold value) is exceeded. Above the threshold value, the severity of disorder increases with the dose. Effects of this type are termed deterministic effects. If biodefensive mechanisms fail to function well in the process of repair of radiation-irradiated cells that have survived, malignancy, ie, cancer, can develop after a latent period. The probability of onset of cancer due to radiation probably rises in proportion to the increase in dose, without a threshold value, at least at doses sufficiently lower than the threshold value for deterministic effects. The severity of cancer is not influenced by dose. Effects of this type are also known as stochastic effects. In the event of such an injury to germ cells, the effects of radiation will be manifested in offspring of the person exposed. Stochastic effects of this type are termed genetic effects.

\section{Measuring Radiation (Radiometry)}

The ionizing radiation (hereinafter simply referred to as radiation) delivered to a patient is mostly absorbed in his or her body tissue, with only a very small portion permeating the body to contribute to the formation of images. In addition, the radiation delivered to the human body scatters in ambient space, reaching the bodies of medical personnel. The radiation delivered to human beings can have adverse effects. Because the amount of exposure can be markedly reduced by implementing appropriate management, however, dosimetry is of critical importance in quantifying the level of exposure of individuals in particular conditions and thus enabling control of exposure and ensuring safe procedures.

\section{(1) Tissue-Absorbed Dose}

The tissue-absorbed dose of radiation is defined as the energy per unit mass of human tissue or organ transmitted by the radiation. As such, the tissue-absorbed dose serves as the basis for calculations of equivalent dose and effective dose, and is also used to express doses that have caused acute radiation injuries and in other cases as well. The international system of units (SI) for tissue absorbed doses is $\mathrm{J} / \mathrm{kg}$, with "gray" and "Gy" used as the special unit name and symbol, respectively. 


\begin{tabular}{|c|c|c|}
\hline Effect & $\begin{array}{l}\text { Approximate } \\
\text { threshold } \\
\text { dose (Gy) }\end{array}$ & Time of onset \\
\hline \multicolumn{3}{|l|}{ SKIN } \\
\hline Early transient erythema & 2 & 2 to 24 hours \\
\hline Main erythema reaction & 6 & Within 1.5 weeks \\
\hline Temporary epilation & 3 & Within 3 weeks \\
\hline Permanent epilation & 7 & Within 3 weeks \\
\hline Dry desquamation & 14 & Within 4 weeks \\
\hline Moist desquamation & 18 & Within 4 weeks \\
\hline Secondary ulceration & 24 & $>6$ weeks \\
\hline Late erythema & 15 & 8 to 10 weeks \\
\hline Ischaemic dermal necrosis & 18 & $>10$ weeks \\
\hline Dermal atrophy (1st phase) & 10 & $>52$ weeks \\
\hline Induration (invasive fibrosis) & 10 & \\
\hline Telangiectasis & 10 & $>52$ weeks \\
\hline Dermal necrosis (delayed) & $>12$ & $>52$ weeks \\
\hline Skin cancer & None known & $>15$ years \\
\hline \multicolumn{3}{|l|}{ EYE } \\
\hline Lens opacity (detectable) & $>1$ to 2 & $>5$ years \\
\hline Lens/cataract (debilitating) & $>5$ & $>5$ years \\
\hline
\end{tabular}

Adapted from ICRP Publication 85: Avoidance of Radiation Injuries from Medical Interventional Procedures, 85, Annals of the ICRP, Vol. 30 No. 2, 2000, with permission from Elsevier Inc.

\section{(2) Equivalent Dose}

Even when the mean tissue-absorbed dose for a tissue or organ is constant, the effects of radiation on living organisms vary depending on the type of radiation. For example, the likelihood of chromosomal abnormality differs between $\mathrm{X}$-rays and neutron rays. Essentially, the equivalent dose of radiation is a modification of the mean absorbed dose for each tissue or organ taking into account the effects of the radiation, and has been defined as an index of the risk of carcinogenesis and genetic effects in tissues and organs resulting from low-dose exposure (risk of stochastic effect). In related laws, however, the equivalent dose is used as an index of the risk of deterministic effects on the skin, lens, and female abdomen (fetuses). The special unit name and symbol for equivalent dose are "sievert" and "Sv", respectively.

\section{(3) Effective Dose}

Susceptibility to radiation-induced cancers and genetic effects varies among tissues and organs. Hence, the equivalent dose may be averaged by weighing according to the radiosensitivity of each tissue or organ to obtain a numerical index known as the effective dose. The effective dose serves as an index of the risk for carcinogenesis and genetic effects resulting from low-dose exposure in individual persons. In related laws, the effective dose is used to specify not only the limits of exposure to persons engaged in medical practice, but also place related limitations, such as the boundaries of radiation-controlled areas. This is because these dose limits have been established as legal regulations on the doses to which persons entering a certain location may possibly be exposed. As with equivalent dose, the special unit name and symbol for effective dose are "sievert" and "Sv", respectively., 2,3

\section{Classification by Severity of Radiation- Induced Skin Injuries and Clinical Course}

If the human body is affected by tissue damage resulting from radiation exposure, this condition is termed a radiation injury. Some types of radiation injuries have no clinical symptoms, and are hence undetectable without appropriate examination. It has been shown that threshold doses exist for deterministic effects, such as skin and lens injuries, under which such effects are not observed. The doses irradiated in ordinary imaging procedures such as chest X-ray radiography are much smaller than the threshold doses and will never cause skin injury. However, in PCI, catheter ablation, and other procedures that can involve the delivery of large amounts of radiation, skin injuries can occur, making confirmation of radiation dose of paramount importance. A list of effects of radiation on the skin and lens, threshold doses, and times of onset is given in Table 1. The threshold dose is a numerical value that must always be borne in mind by the individual responsible for radiation delivery in the context of protection against radiation injuries. In performing PCI or catheter ablation, it is important that the patient's skin-absorbed dose be kept below the threshold value for serious injuries, in order to prevent serious deterministic effects. It should be noted, however, that in actual cases of skin or lens injury, the course is widely variable; the numerical value is thus not applicable to all cases. Prior to performing PCI or similar procedures, the upper limit of the skin dose should be specified as a numerical target for radiation management at the institution. Since there are cases in which priority is given to completion of the treatment over control of minor deterministic effects, as in emergency medical ser-
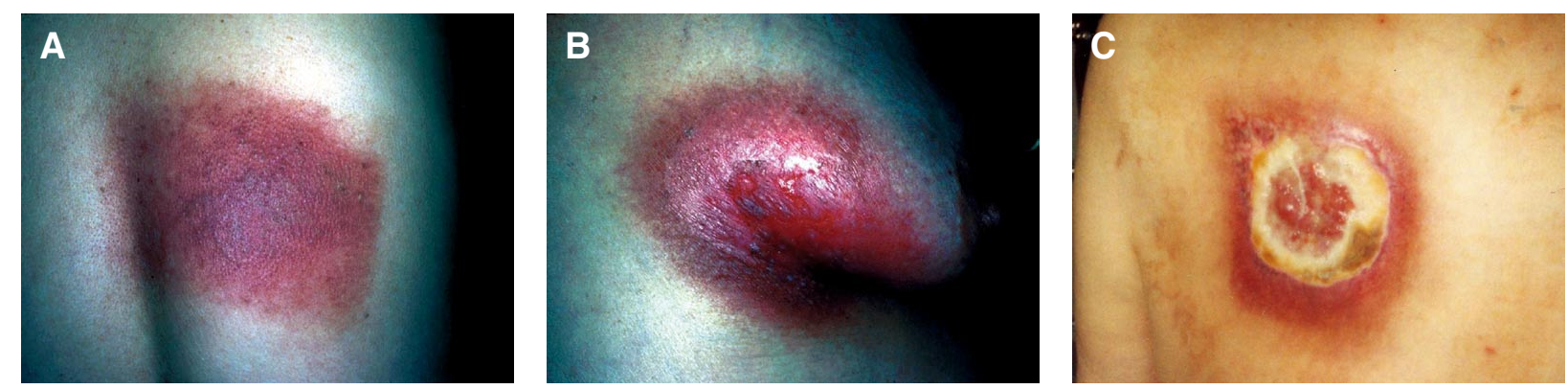

Figure 1. Radiation-induced skin injuries. (A) Dry dermatitis, (B) Moist dermatitis, (C) Ulceration. 
vices, it is necessary to determine in advance the procedural steps to be taken in order to obtain the best outcome for the patient; this includes determination of the individual making judgments regarding continued delivery of irradiation at levels exceeding the management target value.

The earliest change after acute delivery of radiation is transient erythema, which develops in several hours. This is due to capillary dilation upon release of a histamine-like substance from damaged epithelial cells, and is only rarely observable clinically. The skin damage that follows (acute skin reaction) is classified into four grades of severity, from degree 1 to degree 4 , as shown below.

\section{(1) Skin Reactions of First Degree}

After irradiation, the proliferation of epithelial basal cells is first inhibited, followed by keratinized layer desquamation, resulting in thinning of the epithelium. This reaction emerges about 3 weeks after irradiation of 3 to $4 \mathrm{~Gy}$ dose. The skin becomes dry, and epilation occurs. Almost no other symptoms develop.

\section{(2) Skin Reactions of Second Degree [Dry Dermatitis] (Figure 1A)}

The major symptom is main skin erythema, in which arterioles become partially stenosed, with increased blood flow, resulting in dry dermatitis. The skin congests and swells, but does not erode, then desquamation begins. Erythema becomes evident about 2 weeks after irradiation of 6 to $19 \mathrm{~Gy}$, and it persists for about 3 to 4 weeks.
(3) Skin Reactions of Third Degree [Moist Dermatitis] (Figure 1B)

Irradiation of a single dose of 20 to $25 \mathrm{~Gy}$ produces bullae in the epithelium. At higher doses, bullae also appear in subcutaneous tissue, and fuse together. Upon breakage of the bullae, subcutaneous tissue becomes bare. Moist dermatitis develops about 1 week after irradiation and persists for 4 to 5 weeks. Fibrin deposits in the wounds. Affected regions are susceptible to infection. After about 1 to 2 weeks regeneration of the epithelium begins.

\section{(4) Skin Reactions of Fourth Degree [Ulceration] \\ (Figure 1C)}

These reactions occur within 1 week after irradiation of a dose of $30 \mathrm{~Gy}$ or more. Deep-red erythema develops, followed by formation of bullae, which in turn erode to form ulcers, and the epithelium necrotizes and sloughs off. At higher doses, sharply indented radiation ulcers typically develop. The epithelium loses its basal membrane, leaving the thin layer of epithelium directly in contact with subcutaneous tissue; the affected skin is now vulnerable to mechanical stress.

It is important that the dose received by the patient in PCI be accurately determined with the above facts in mind, and that the dose and time of onset of its effects be confirmed. Whenever possible, the patient should be followed for an appropriate period, considering the time course of the adverse reactions to radiation described above. ${ }^{4-9}$

\section{Specific Issues (Qs and As)}

\section{Basic Knowledge of Radiation-Induced Skin Injuries}

\section{A Radiation-Induced Skin Injuries in PCI}

Q1: I've heard that in $\mathrm{PCl}$ the patient receives higher doses than in other radiological procedures. Is this true?

A: Figure 2 shows mean cumulative doses per exam in 62 patients undergoing PCI and diagnostic contrast-enhanced coronary angiography (CAG) at the National Cardiovascular Center. It is evident that PCI involves greater doses than diagnostic contrast-enhanced CAG. In PCI, the transillumination time is extended because a catheter must be inserted into the target coronary artery, and a thin guidewire, balloon,



Figure 2. Patient exposure doses in diagnostic coronary angiography and percutaneous coronary intervention (PCI). Comparative data with 1 unit dose received by the patient in conventional diagnostic coronary angiography. and stent are inserted into the coronary artery and dilated or left deployed. Imaging must be repeatedly performed to check the positions and degrees of patency of the balloon and stent. As a result, the dose received by the patient increases. ${ }^{9}$

Q2: I've heard that in $\mathrm{PCl}$ greater exposure occurs during transillumination than during imaging. Is this true?

A: In diagnostic contrast-enhanced angiography, catheter insertion in the target coronary artery is followed only by repeated positioning and imaging in various directions. Since PCI, on the other hand, involves not only catheter insertion

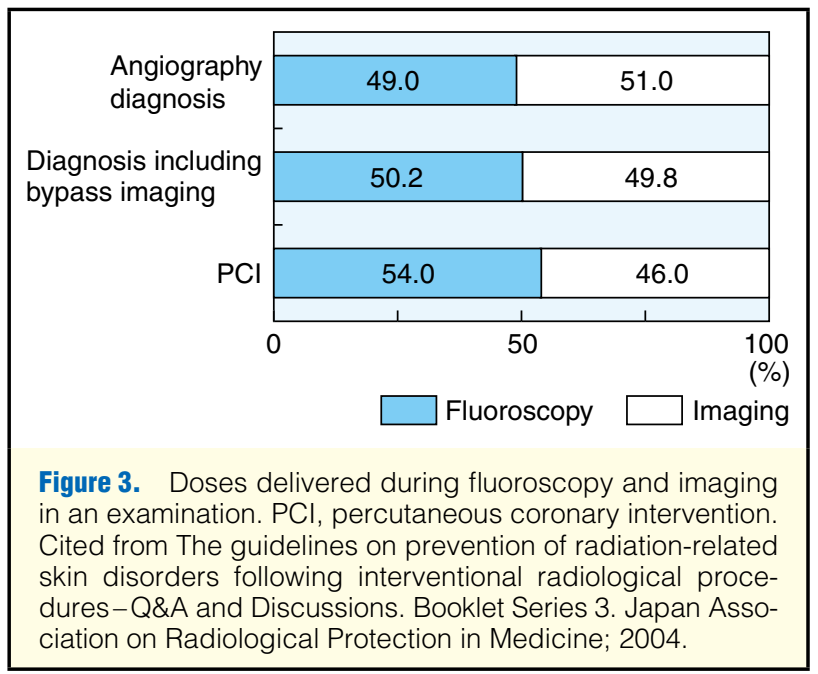




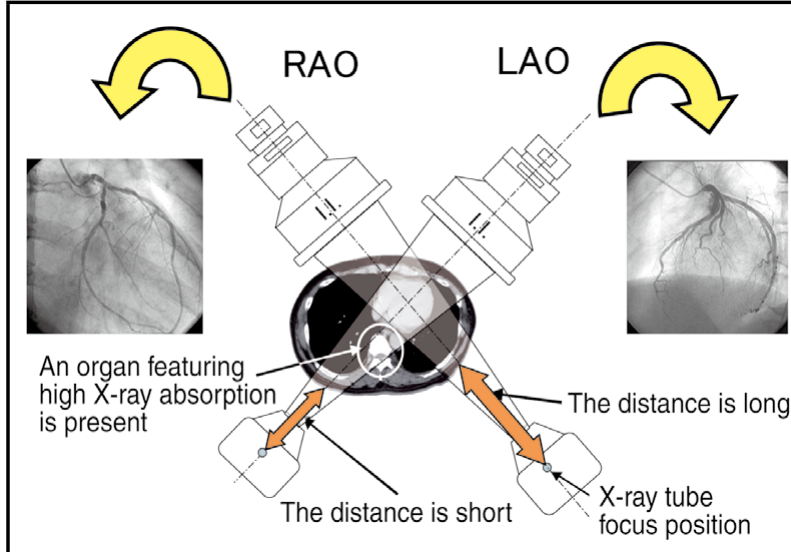

Figure 4. Differences in X-ray entrance angle and exposure dose between $\mathrm{LAO}$ and RAO. LAO, left anterior oblique; $\mathrm{RAO}$, right anterior oblique; I.I., image intensifier.

into the target coronary artery but also the delivery of a thin guide wire, balloon, and stent to the inside of a coronary artery and dilation and positioning of the stent at deployment, transillumination time must be extended. In addition imaging is repeatedly performed to check the positions and degrees of dilation of the balloon and patency of the stent. Figure 3 shows mean transillumination - imaging dose ratios per exam in 62 patients undergoing CAG at the National Cardiovascular Center. Although PCI involves longer transillumination times than diagnostic contrast-enhanced angiography, the number of times imaging is performed increases proportionately, so the ratio of doses required for transillumination and imaging does not differ much between PCI and CAG performed for diagnostic purposes. ${ }^{4}$

Q3: How many grays of exposure are needed to induce complications such as skin erythema and skin ulcers on a patient?

A: The human body is influenced by radiation in various ways. If the skin-absorbed dose is $\mathbf{2} \mathbf{G y}$ or more, transient erythema of the skin can occur relatively early (within several hours), while irradiation of $\mathbf{2 4 G y}$ or more can cause skin ulcers (see Table 1)., ${ }^{4}$

\section{B Areas Where Radiation-Induced Skin Injuries Are Most Likely to Occur}

Q4: Why are skin injuries more prevalent on the right part of the back in $\mathrm{PCl}$ ?

A: As shown in Figure 4, the distance between the X-ray tube focus and the patient's skin surface is shorter in the left anterior oblique (LAO) position than in right anterior oblique (RAO) position because the heart is located on the patient's left side. Even with delivery of X-irradiation of the same intensity for the same length of time, a greater dose enters the patient's body in LAO than in RAO. When the direction of X-ray entrance is RAO, the heart is observed through the left lung from the patient's left back. In contrast, in the case of LAO, the heart is observed through the vertebral column and mediastinum from the patient's right back. The lungs are easily permeated by X-rays because they contain much air, while the vertebral column and mediastinum are much less permeable to X-rays because of their high densities, and thus requiring higher doses. For this reason, skin injuries are more prevalent on the right part of the patient's back than elsewhere. ${ }^{9}$

\section{Informed Consent and Countermeasures in Case of Excess Exposure and Onset of Radiation-Induced Skin Injuries}

\section{A Matters to Be Included in the Explanation \\ of Radiation Injuries}

Q5: In obtaining informed consent prior to CAG or $\mathrm{PCl}$, what should be explained regarding radiation injuries? I am concerned that explanation of such injuries may increase patient anxiety.

A: Although the PCI procedure is less invasive than ordinary surgical operations, it involves the use of radiation, so prolonged treatment can result in radiation-induced skin injuries. It is therefore important that prior to performing PCI an explanation of radiation-induced skin injuries be provided, in addition to information on the method used in the procedure and possible complications.

Since inconsistency in content of explanations among those providing them makes the patient more anxious, it is important that an explanatory manual or the like be produced to ensure that the contents of explanation are unified across the institution. Although expert knowledge is required to explain the effects of radiation, it is important that the provider of the explanation bear in mind the reason for the patient's anxiety, rather than merely using jargon and presenting numerical data, so as to put him or her at ease.

Since a specific explanation helps ease the patient, the operator should endeavor to be able to explain the following:

- There are threshold values for radiation-induced skin injury.

- The dose can exceed the threshold value for radiationinduced skin injury depending on the course of treatment. In such cases, consent to continue or discontinue the examination is to be obtained from the patient.

- The system used for delivery of radiation is appropriately controlled to ensure that the examination is always performed at the optimal dose.

- The operator will endeavor to check skin entrance doses by monitoring the conditions of irradiation and implementing other protective measures.

- Countermeasures against radiation-induced skin injury are available.

Explanation of the above is required even in emergency examinations. ${ }^{9,10}$

\section{B Explanation and Consent During PCI Procedure and Subsequent Measures}

Q6: If transillumination time is prolonged during $\mathrm{PCl}$ to the extent that the patient exposure dose approaches levels that can cause skin injuries, how can I determine whether to continue or discontinue the procedure?

A: The threshold dose for possible onset of early transient erythema is $\mathbf{2} \mathbf{G y}$ (Table $\mathbf{1}$ ). The exposure dose per unit time varies widely among different institutions, and also depends on imaging conditions (the patient's body type, angle of imaging, frame rate, etc). Therefore, each institution should establish a reference level of transillumination and imaging times corresponding to $2 \mathrm{~Gy}$. When it is found that the sum of transillumination and imaging times is likely to reach this level, the operator should determine whether to continue or discontinue the examination or procedure. If the operator 
A

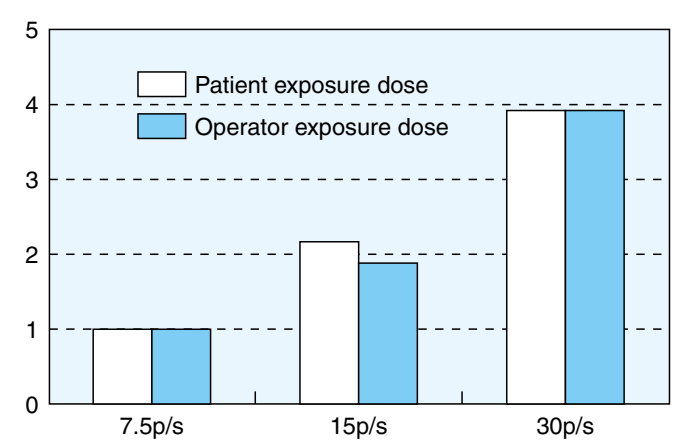

B

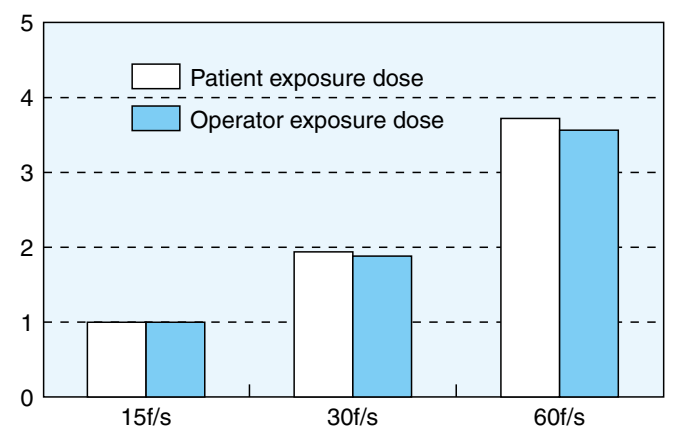

Figure 5. (A) Relationship between pulse rate and exposure dose in fluoroscopy. Exposure doses are expressed using the dose at $7.5 \mathrm{p} / \mathrm{s}$ as a unit (using 9-inch image intensifier and acrylic resin phantom $20 \mathrm{~cm}$ in diameter). (B) Relationship between number of imaging frames and exposure dose. Exposure doses are expressed using the dose at 15f/s as a unit (using 9-inch image intensifier and acrylic resin phantom $20 \mathrm{~cm}$ in diameter).

judges that the benefits to the patient of continuing the examination or treatment will outweigh the risk of radiation injuries, the examination or treatment may be continued. It is desirable, however, that even when prior consent has been obtained with a full explanation of the risk of radiationinduced skin injury, the patient's intent be reconfirmed at the time when this judgment is made. If it is difficult to confirm the patient's consent, consent may be obtained from his or her family. In situations in which the patient's life is threatened by acute myocardial infarction, shock, or other severe conditions, priority should be placed on life-saving rather than on avoiding the onset of radiation-induced skin injuries.

If the examination or procedure is continued even after the threshold dose is judged to have been reached, still greater caution should be exercised in reducing the exposure dose. Possible measures include (1) lowering the fluoroscopy pulse rate and/or imaging frame rate and (2) changing the fluoroscopy and/or imaging angle to shift the skin irradiation field to other positions (see Q22 and Figure 15).

\section{Explanation of and Measures Taken After Exposure to Excessive Doses}

Q7: If it is found after the performance of catheter intervention that the patient exposure dose may have exceeded the threshold dose for the onset of skin injury, what measures should be taken?

A: The person in charge of radiation safety management should inform the attending physician of the predicted dose and severity of skin injury, and request subsequent countermeasures.

The specific actions to be taken are as follows:

1. Obtain informed consent from the patient for such actions (see Q8).

2. Prepare a patient skin-absorbed dose report and inform all persons concerned of the delivery of a dose exceeding the threshold as documentation for follow-up examination (see Q9).

3. Detection of initial injuries: Early transient erythema appears soon after the examination, ask an attending physician or nurse to monitor the site of irradiation.

4. Notification to dermatologist and request for cooperation (if required because of severity of predicted injury): Notify the dermatologist of the site of irradiation, exposure dose, and predicted severity of skin injury. It is desir- able that the skin-absorbed dose report be submitted with attachment of an examination status report (examination records) and reference documents concerning radiationinduced skin injuries.

With these contents included, a manual should be prepared for the institution in order to deal with skin injuries. In all cases, it is important that all staff members endeavor to develop and maintain good communication with each other in order to work together as a team. ${ }^{9,10}$

Q8: If a dose that can cause radiation-induced skin injuries has been delivered, what explanation should the patient receive?

A: Irrespective of whether prior informed consent has been obtained, inform the patient that the treatment was necessary, and provide an explanation of your institution's policy on the treatment of skin injuries. Even when prior informed consent has been obtained, an explanation should be provided again for the sake of confirmation.

The patient should be given the following advice and suggestions regarding measures to be taken for the parts of the skin where injuries may occur:

1. Periodic medical follow-up examination is necessary.

2. The effects of radiation usually appear after a time lag.

3. Tell the patient the parts of the skin where injury may occur, and instruct him or her not to scratch them, to avoid the use of highly irritating bathing agents and soaps during bathing, and not to apply any drugs other than those prescribed by the physician.

4. If any change occurs in skin symptoms, the patient should undergo medical examination.

Q9: If a dose that can cause skin injury has been delivered, is it necessary to record the fact? If so, please show how this is done and which form is used.

A: In PCI performed with repeated delivery of radiation, the effects of radiation are additive, even doses lower than the threshold value can cause skin injury, and repeated irradiation during a procedure can exacerbate an injury. In case of irradiation to the same site with short intervals between bouts of irradiation, the site of irradiation and dose should be recorded, and the operator should strive to prevent excessive irradiation. In the International Commission on Radiological Protection 


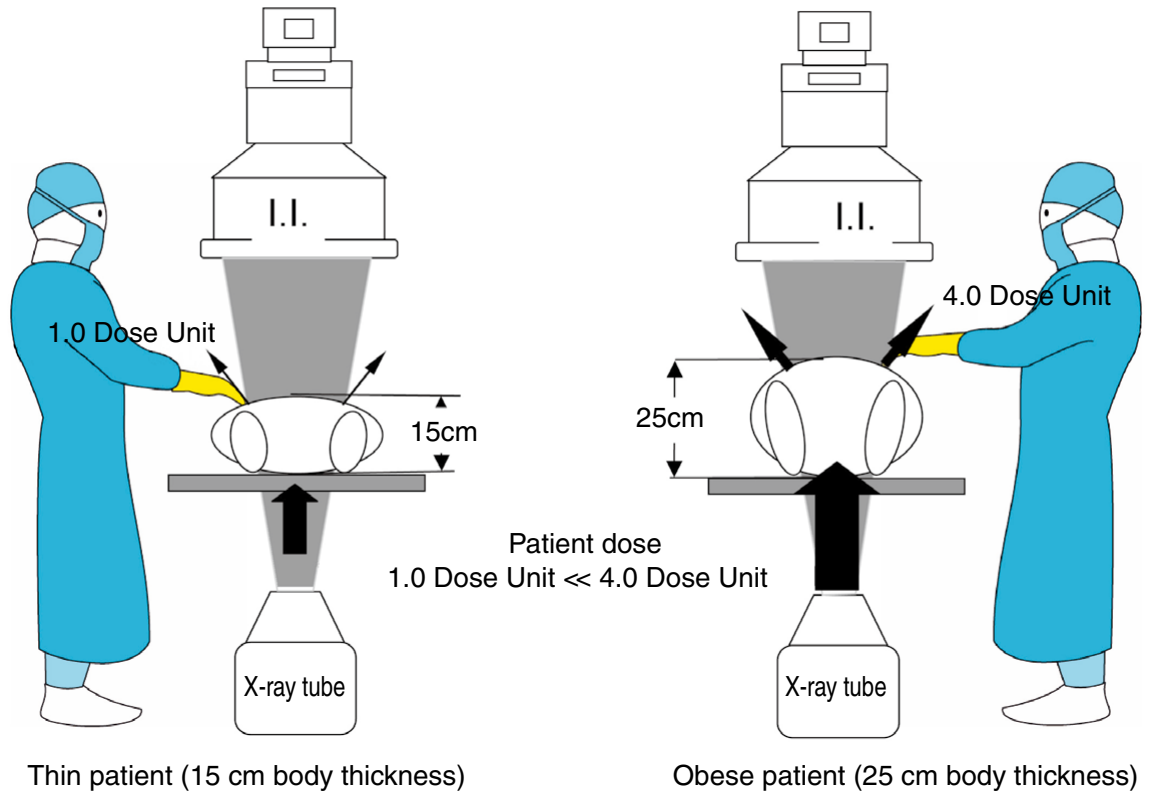

Figure 6. Relationship between patient body type and exposure dose. Doses received by the patient/operator are expressed relative to those in the thin patient case, with the latter considered 1 dose unit (7.5-inch image intensifier [I.I.], pulsed fluoroscopy at $15 \mathrm{p} / \mathrm{s}$ ).

(ICRP) Publ.85, it is recommended that if the skin exposure dose can be estimated to be $3 \mathrm{~Gy}$ ( 1 Gy for cases of repeated irradiation) or more, the estimated dose and the site of irradiation be indicated on an appropriate body surface map.

\section{Measures Taken in Case of Onset of Radiation-Induced Skin Injuries}

Q10: In case of slight acute dermatitis that has remitted quickly, is it necessary for the patient to be treated with medication or to undergo ambulatory treatment at an outpatient dermatology clinic?

A: If slight skin injury develops early after performing PCI and then disappears spontaneously, the condition is deemed early transient erythema (early erythema: threshold dose $=$ $2 \mathrm{~Gy}$ ) or main erythema (threshold dose $=6 \mathrm{~Gy}$ ). Main erythema often leaves pigmentation (or depigmentation) after healing. If it has healed without pigmentation, it is quite unlikely that delayed skin injury will develop later, so no dermatological treatment is necessary, nor is there any need for periodic follow-up examination. Visits to a dermatologist are needed only after the patient or his or her family has noted a change in the patient's skin. If an dyschromia remains in a part of the skin where rash has healed (main erythema), the risk of subsequent development of delayed skin injury cannot be ruled out even if the change is initially very mild, so periodic follow-up examination by a dermatologist is required. Regarding the duration and frequency of ambulatory medical checkups, it is desirable that the patient visit the institution every 3 months for about 1 year. Even if the dyschromia remains, however, macroscopic observation alone is sufficient, with no special treatment required. In all cases, it is important that the site of irradiation and dose be recorded at the time of irradiation. ${ }^{9}$

\section{Variables Affecting Exposure Doses}

\section{A Effects of Irradiation Pulse Rate}

Q11: To what extent can I reduce the exposure dose by lowering the pulse rate or image acquisition rate during fluoroscopy?

A: Pulsed fluoroscopy is effective as a method of reducing exposure in PCI. As shown in Figure 5A, exposure dose decreases as the pulse rate falls from $30 \mathrm{p} / \mathrm{s}$ to $15 \mathrm{p} / \mathrm{s}$ and then to $7.5 \mathrm{p} / \mathrm{s}$. It should be noted, however, that the doses irradiated at high pulse rates are similar to those with continuous fluoroscopy, so if pulsed fluoroscopy is used in an attempt to reduce patient exposure, a low pulse rate must be used. In the system shown in Figure 5A, there is a proportional relationship between pulse rate and dose. In other type of systems as shown in the figure, however, even when a low pulse rate is chosen to examine a patient with a large body, the system automatically expands the pulse width, increases the tube current, or takes other measures to ensure that doses comparable to those with high pulse rate fluoroscopy are delivered. In such cases, choosing a low pulse rate does not always lead to a reduced exposure dose. Prior to using the system, it is necessary to become familiar with the its performance by taking actual measurements using it, or asking the manufacturer to provide detailed specifications for the instrument in different operating conditions. In addition, since it is difficult to confirm the position of catheters and other devices in blood vessels under low pulse rate fluoroscopy if performed by an unskilled operator, it is important that this technique be utilized only after a full discussion with the operator.

The image acquisition rate during imaging also influences patient exposure dose. Figure 5B shows the doses received by the patient at image acquisition rates of $15 \mathrm{f} / \mathrm{s}, 30 \mathrm{f} / \mathrm{s}$, and $60 \mathrm{f} / \mathrm{s}$. Because the dose received by the patient increases as 


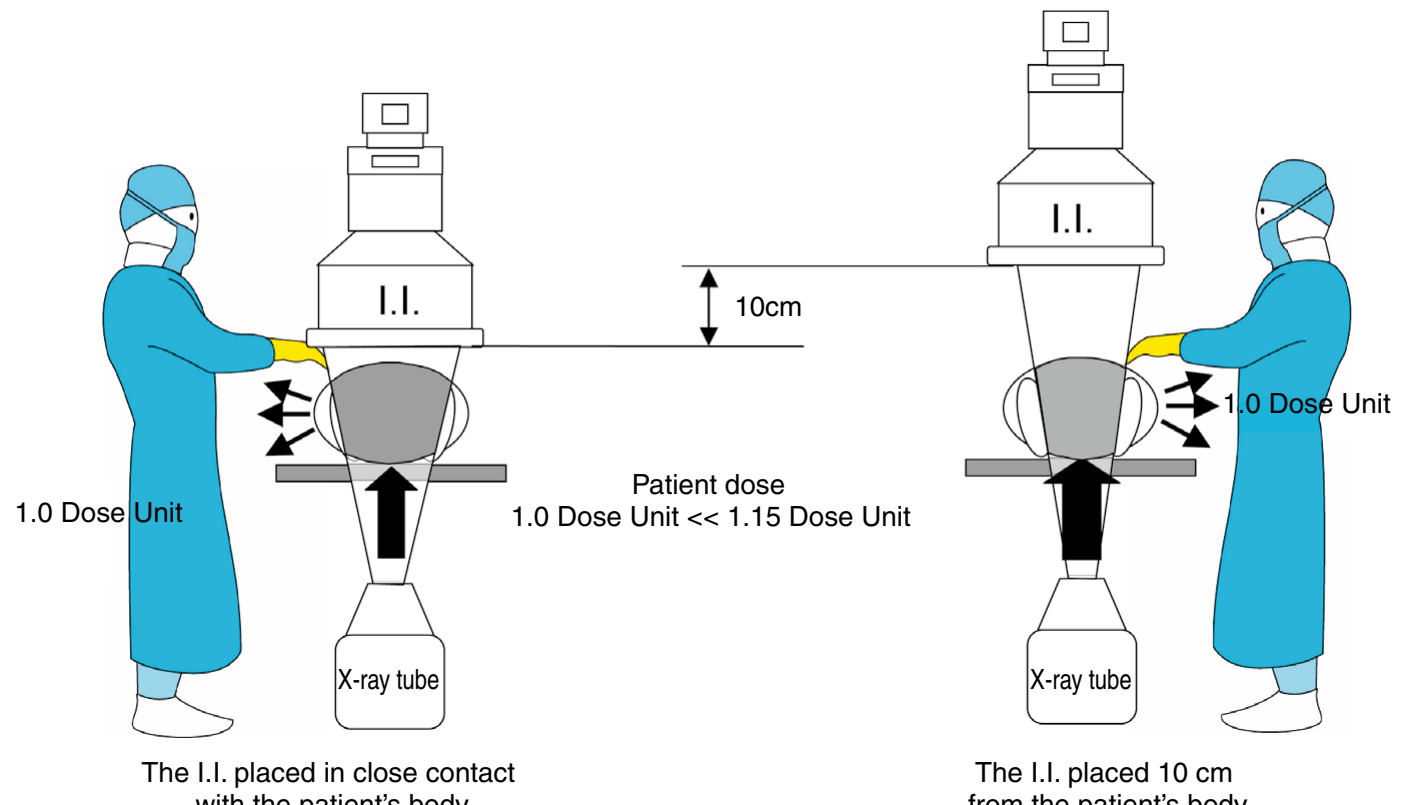

Figure 7. Relationship between image intensifier (I.I.): subject distance and exposure dose. Comparative data with 1 dose unit received by the patient/operator with the I.I. in close contact with the patient's body.



Figure 8. Relationship between exposure dose and patient - X-ray tube focus distance. When the distance between the X-ray tube and patient is increased by $10 \mathrm{~cm}$, the dose received by the patient decreases by $13 \%$. I.I., image intensifier.

the image acquisition rate rises, emphasis should be placed on choosing an image acquisition rate suitable for the patient's heart rate and pathologic condition, and minimizing the dose received by the patient, rather than on increasing the image acquisition rate merely to improve visibility. ${ }^{11}$

\section{B Effects of Patient Body Type}

Q12: To what extent does exposure dose differ depending on the body type of the patient?
A: Generally, obese patients receive greater doses per unit time than thin patients. Figure 6 shows the difference in entrance surface dose for two patients $15 \mathrm{~cm}$ and $25 \mathrm{~cm}$ in body thickness; as the subject of imaging thickens by $10 \mathrm{~cm}$, the entrance surface dose nearly quadruples. Therefore, when the subject is an obese patient, the cumulative dose administered during examination must be carefully monitored in order not to administer excessive doses of radiation. Of note, the operator is also subject to greater exposure when 


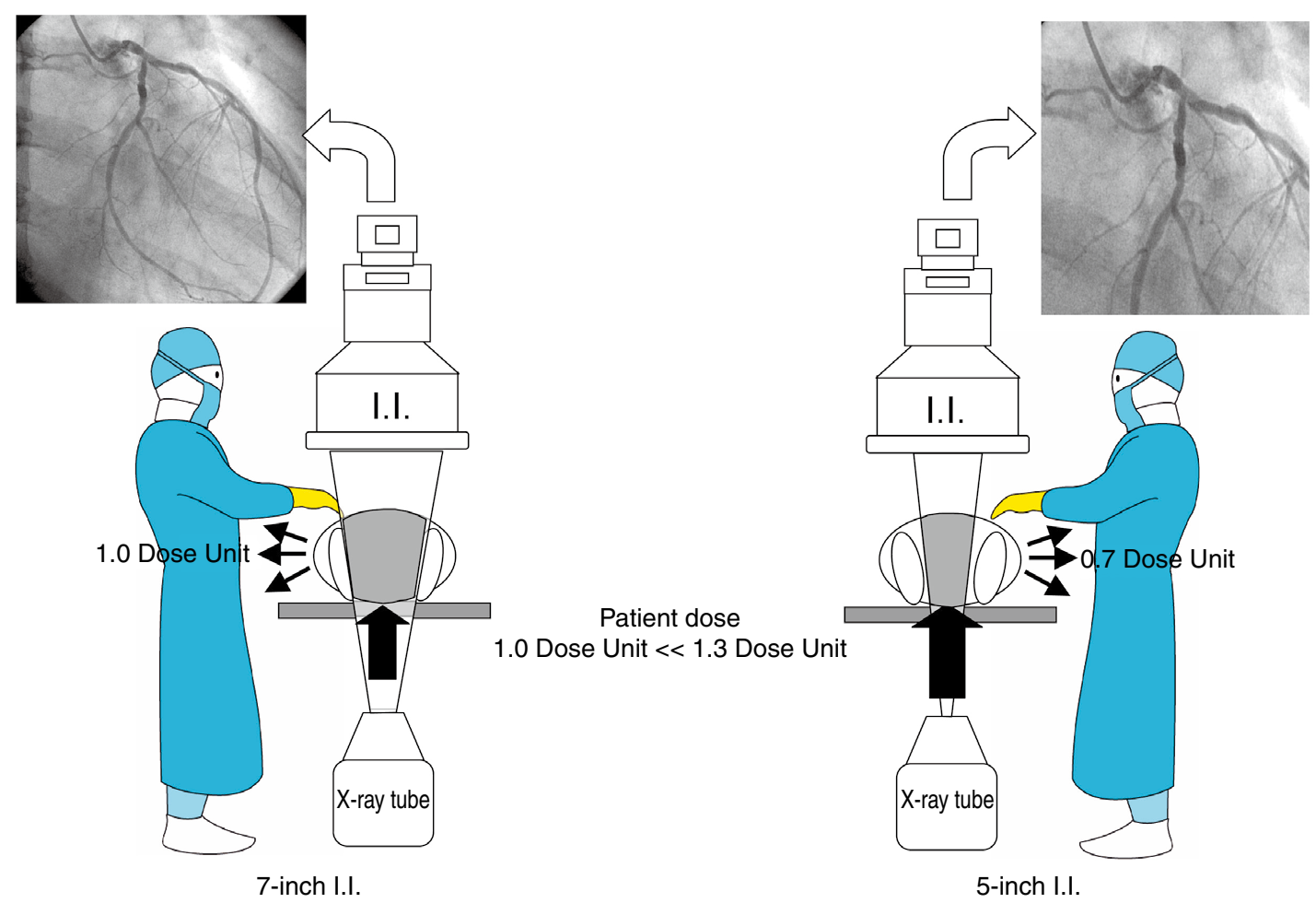

Figure 9. Magnification in fluoroscopy/imaging and exposure doses. Comparative data with 1 dose unit received by the patient/operator from a 7-inch image intensifier (I.I.).

examining an obese patient.

\section{Distance Between Image Intensifier and Patient}

Q13: Why does the exposure dose increase as the image intensifier becomes more distant from the patient? What is the operator exposure dose under such conditions?

A: If the distance between the X-ray tube and patient is constant, the distance between the X-ray tube and image intensifier increases as the image intensifier becomes more distant from the patient, so automated adjustment by the system ensures delivery of a larger amount of X-rays. As a result, the patient exposure dose increases. Figure 7 shows the change in entrance surface dose where radiation enters when the image intensifier is distanced from the patient while keeping the catheter table height constant. With increase in distance of the image intensifier of only $10 \mathrm{~cm}$, the entrance dose increases by about $15 \%$. In routine examination, the image intensifier and patient are often separated by about $10 \mathrm{~cm}$. However, since even small changes will lead to major differences in dose if they accumulate, the distance between the image intensifier and the patient must be kept as close as possible in order to avoid excessive irradiation of the patient. The dose received by the operator changes relatively little when the image intensifier is placed more distant from the patient. ${ }^{9}$

\section{Distance Between X-Ray Tube and Patient}

Q14: Tips for reducing radiation exposure include the statement "distance the patient from the X-ray tube as much as possible." Why? And what is the operator exposure dose when the examination table is at low position?

A: As the distance of the patient from the X-ray tube is increased, the distance between the X-ray tube focus and image intensifier increases, so the X-ray output increases. At the same time, however, the amount of low-energy X-rays reaching the patient, which do not contribute to images but do have a major impact on patient exposure, decreases, so skin dose decreases. This is exemplified in Figure 8. As the patient is brought about $10 \mathrm{~cm}$ closer to the X-ray tube, the dose increases by about $15 \%$, so it is necessary to heighten the catheter table to distance the patient from the X-ray tube, as long as this does not interfere with procedures by the operator. The dose received by the operator remains unchanged with this alteration.

When a short operator performs the examination, he or she is apt to lower the catheter table to facilitate the operation. However, lowering the table makes the patient and $\mathrm{X}$-ray tube approach each other, which in turn increases the patient exposure dose; caution thus needs to be exercised in this regard. ${ }^{9}$

\section{E Size of Transillumination Field}

Q15: In PCl, magnified views are often used to obtain clear images of the guidewire and stent. What is the extent of change in dose received by the patient associated with this?

A: When the image intensifier size is reduced and the screen is expanded, the dose received by the patient increases, as shown in Figure 9. By contrast, when the image intensifier size is increased and the field is widened, the dose decreases. Traditionally, this has not commonly been performed since magnified views with smaller image intensifier sizes lead to a lack of dose, which hampers obtaining clear images. However, 


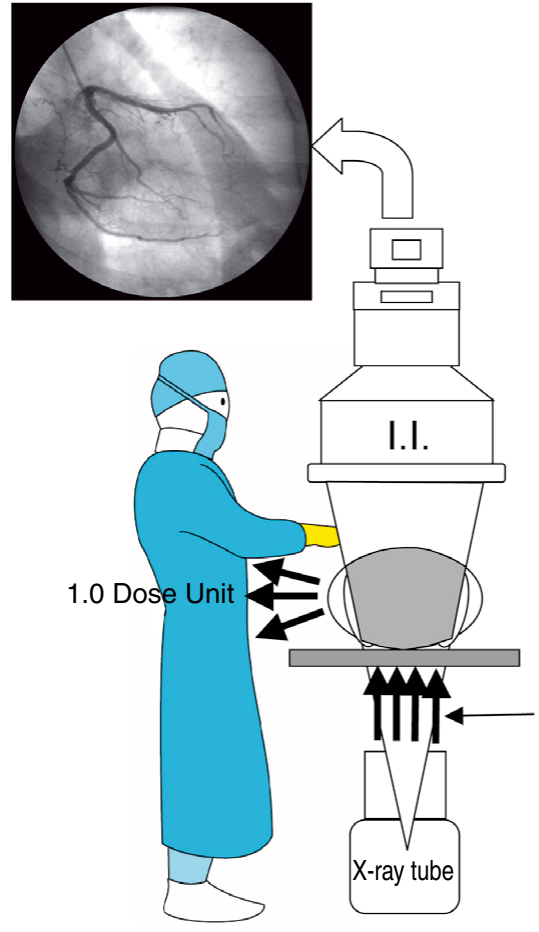

Fully open irradiation field

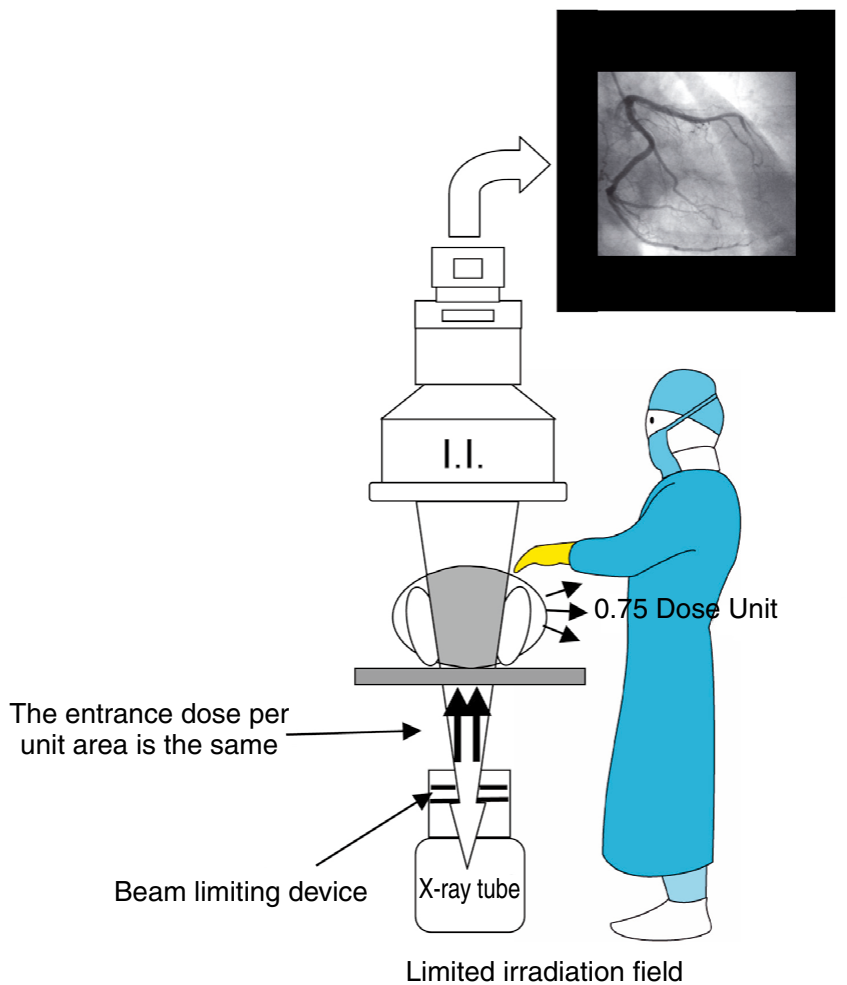

Figure 10. Beam limiting device and exposure dose. Comparative data with 1 dose unit received by the operator when the field of irradiation is fully open (7-inch). I.I., image intensifier.

recent technical innovations, including the expanded capacity of X-ray tubes and the introduction of new technologies such as digital image processing have, along with the spread of PCI, led to the current wide use of magnified views at many institutions. Although this is essential for ensuring safe performance of PCI, it results in greater patient doses, so use should be limited to the minimum required frequency to prevent skin injuries. Even when the field of irradiation is expanded, irradiation of parts of the skin where irradiation is unnecessary must be avoided by using the beam limiting device.

As the image intensifier size is reduced, the field of irradiation is automatically narrowed, and the operator's dose therefore decreases. As the image intensifier size is increased, the field of irradiation widens, with increase in scattered dose and a tendency for operator exposure dose to increase. ${ }^{9}$

\section{F Effects of Beam Limiting}

Q16: As the field of irradiation is narrowed, does patient exposure dose actually decrease?

A: Figure 10 compares patient exposure doses produced with a fully open field of irradiation and a narrowed field of irradiation with $70 \%$ of the initial area. Even when the field of irradiation is narrowed, the dose per unit area received by the patient remains unchanged. However, as the field of irradiation increases, the area of skin at risk for skin reactions to radiation such as ulcers will increase, so effort is always needed to avoid irradiation of sites where it is unnecessary, in order to prevent radiation injury. Additionally, by narrowing the field of irradiation, the area of skin irradiated repeatedly during fluoroscopy or imaging at different angles can be narrowed. In Q22 a method is described for avoiding skin inju-

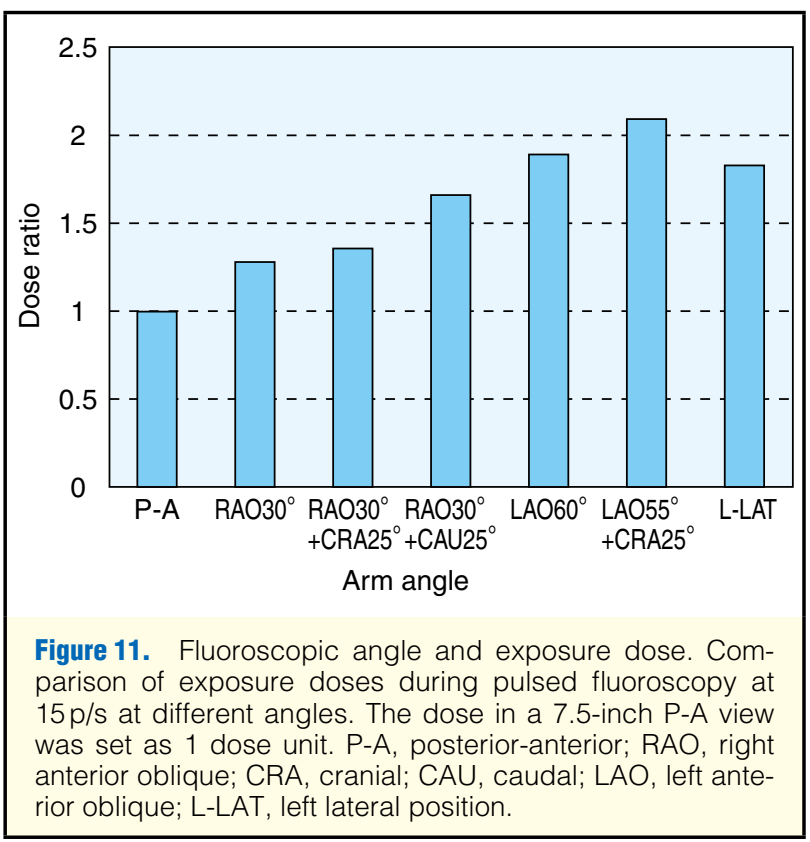

ries by changing the angle of X-ray entrance. When the field of irradiation is limited to a small area in advance, the operator may change the entrance angle only slightly to avoid irradiating the same region of the skin. Of course, the operator's dose also decreases as the field of irradiation is narrowed. ${ }^{9}$ 

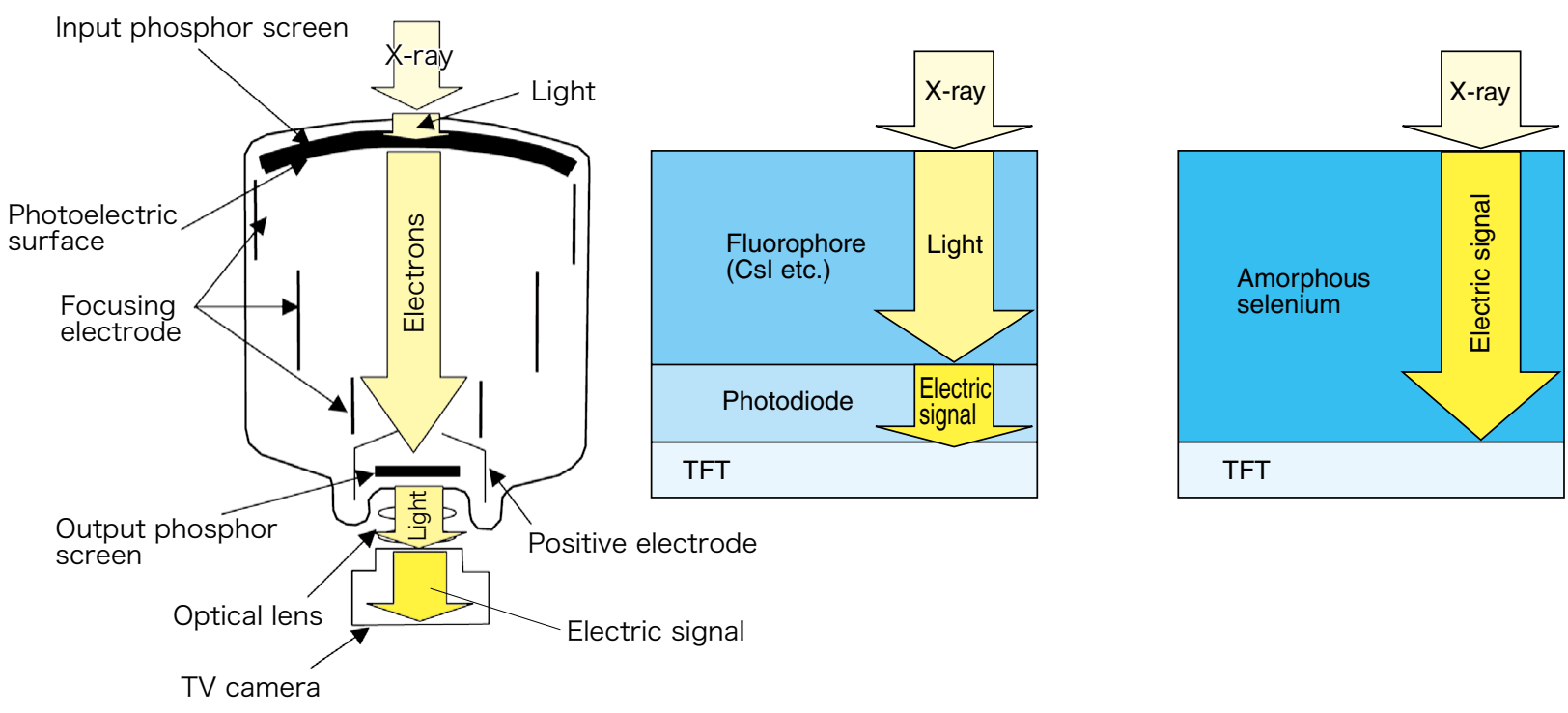

(a) Image intensifier-TV camera type

(b) Indirect conversion type FPD

(c) Direct conversion type FPD

Figure 12. Schemes of flat panel detector (FPD) type imaging systems. Comparison of different types of devices. Csl, cesium iodide; TFT, thin-film transistor.

\section{G Effects of Fluoroscopic/Imaging Angle}

Q17: I've heard that in LAO cranial views and LAO caudal views, the patient skin-absorbed dose is high. Why? And how can I reduce this dose?

A: The X-ray fluoroscopic/imaging device is controlled to keep the dose entering the image intensifier constant. As the direction of X-ray entrance is changed, the thickness of the subject imaged changes as well, and the dose is adjusted according to the thickness irradiated. This is why the patient entrance dose differs depending on the X-ray entrance angle. Figure 11 compares doses with various entrance angles using phantoms; there is an approximately two-fold difference in dose among the different entrance angles evaluated. In the LAO projection, the heart is examined through the vertebral column and mediastinum from the patient's right back, so greater doses are delivered. In the cranial or caudal projection, body thickness increases still more, so greater doses are delivered (see Figure 4). This is the reason why the dose increases in the LAO cranial and LAO caudal projections. When the examination is performed using these X-ray entrance angles, the operator should endeavor to reduce the dose by, for example, lowering the pulse rate or increasing the image intensifier size.

Since PCI involves repeated cycles of fluoroscopy and imaging with a constant X-ray entrance angle for a prolonged period of time, it is important to understand the relationship between the entrance angle and dose for the system used, and to prevent the patient from receiving excessive doses. ${ }^{12}$

\section{H Flat Panel Detector (FPD) Type Imaging System}

Q18: The FPD type imaging system reportedly generally enables reduction of exposure dose, although l've heard that the exposure dose in fact increases in some cases. In which cases does the exposure dose rise? And what are the points of note in using the system?

A: Currently, there are two modes of imaging by FPD: direct conversion and indirect conversion (Figure 12). The direct conversion type employs amorphous selenium (a-Se) as the $\mathrm{X}$-ray imaging medium. The electric charge generated by $\mathrm{X}$-rays in the a-Se medium is directly read out using a thinfilm transistor (TFT). The direct conversion type involves only slight loss of energy during energy conversion, and has excellent spatial resolution. In the indirect conversion type, $\mathrm{X}$-rays entering the imaging system are converted to visible light through a fluorophore such as cesium iodide (CsI) as the X-ray imaging medium, using a photodiode or the like, which is then converted to electric signals. Although some loss of image quality due to light scattering is unavoidable, this type is now commonly used for cardiovascular imaging because it is easy to perform.

Advantages of FPD include the following:

1. The device undergoes less time-related deterioration, ensuring image quality that is stable over a long period of time;

2. Lack of distortion;

3. High contrast.

Since FPD has higher efficiency in X-ray detection than image intensifiers, it theoretically enables reduction of dose. In fact, in imaging at a high dose rate, which is not influenced by X-ray quantum noise (noise caused by random fluctuation of quantum flow), some extent of dose reduction is possible. On the other hand, in low-dose fluoroscopy, which is influenced by X-ray quantum noise, FPD contributes less to dose reduction than image intensifiers, so the cumulative dose per examination is the same as with image intensifiers. However, because FPD has advantages including little time-related deterioration, as well as ease of system quality control and freedom from image distortion, it is expected to replace image intensifiers in the future; expansion of its use and improvement of its performance will together lead to reductions of dose. 

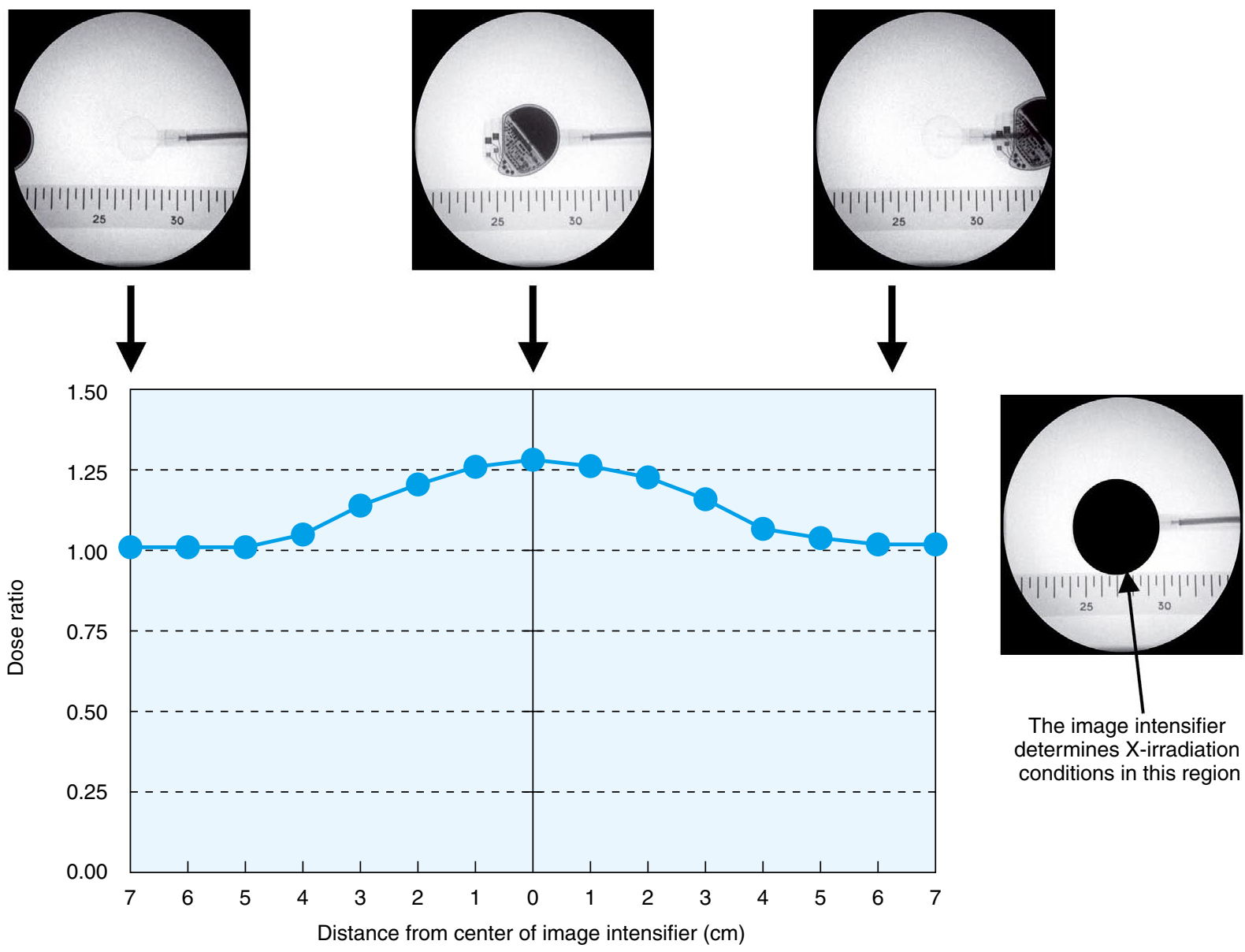

The image intensifier determines $\mathrm{X}$-irradiation conditions in this region

Figure 13. Effects of a pacemaker in the irradiation field on dose. Comparative data with 1 dose unit without pacemaker

In addition, since FPD has a broad dynamic range, no halation is produced even without use of a compensating filter. Another advantage of the FPD device is its ease of use, since the dose does not increase much even when the field is expanded, as with image intensifiers. However, its dynamic range is wide enough that excessive doses of irradiation are likely to be overlooked because of the absence of hindrance to image reconstitution. Even more effort is thus needed to reduce radiation exposure using conventional dose-reduction approaches. ${ }^{13-16}$

\section{Effects of Pacemakers and Leads}

Q19: Why does the exposure dose increase when the body of a pacemaker or a lead enters the field of irradiation?

A: X-irradiation conditions during fluoroscopy and imaging depend on the dose received by the photoreceptor in the center of the image intensifier. Figure 13 shows the relationship between the position of the pacemaker in the field of irradiation and the corresponding dose ratio. When a pacemaker is present in the peripheral portion of the field, where determination of X-irradiation conditions is not affected, the conditions remain unchanged, so the dose received by the patient does not change. However, when the pacemaker is present in the center of the field, X-irradiation occurs in amounts that depend on the material of the pacemaker. Generally, since the pacemaker is made of metal, larger amounts of X-rays are required than for a human body of the same thickness. Therefore, when the pacemaker is present in the center of the field of irradiation, the dose received by the patient increases, and at the same time an excessive dose is delivered to the surrounding tissue where the pacemaker is absent, which can result in deterioration of image quality due to halation or other factors. For these reasons, it is important not to locate the pacemaker in the center of the image intensifier whenever possible.

\section{$J$ Effects of the Upper Arm in the Irradiation Field on Fluoroscopy/Imaging}

Q20: In case of entry of the patient's arm (upper arm) into the irradiation field, does the dose received by the patient change? What are the points to note in protection against skin injury in this case?

A: When X-rays are delivered to a patient for diagnostic or therapeutic purposes, a basic rule of X-ray imaging is to remove objects that interfere with imaging from the irradiation field in order to obtain clear images. For this reason, the patient is asked to raise his or her arm if it enters the irradiation field during coronary imaging. Recently, however, CAG has often been performed with approach from the cubital artery or radial artery, and the number of cases of inability to raise the arm has correspondingly increased.

Additionally, catheter ablation is sometimes performed 


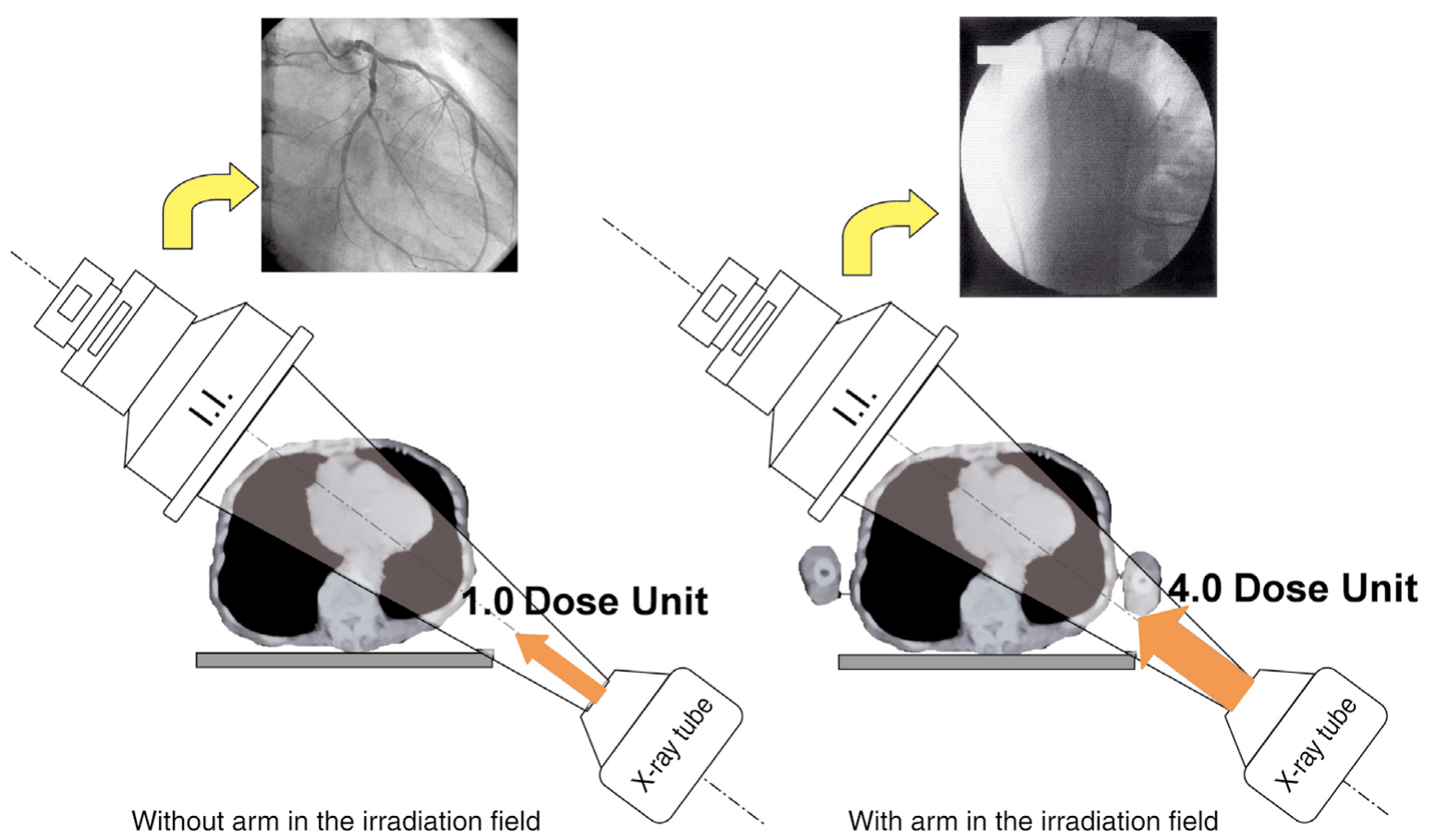

Figure 14. Relationship between patient arm position and exposure dose. Comparative data with 1 dose unit received by the patient without the patient's arm in the irradiation field. I.I., image intensifier.

while neither arm is raised, for various reasons, including the great burden placed on the patient by the long period of examination; the use of an electrode catheter, which features relatively high X-ray visibility, ensuring that the presence of interfering shadows of arms in the field of irradiation does not markedly interfere with examination; and the increased

Table 2. How to Reduce the Patient Dose During Percutaneous Coronary Intervention

1. Avoid unnecessary fluoroscopy and imaging.

2. Set the frame rate to as low a level as possible and shorten the imaging time to minimize imaging-related irradiation.

3. Have a good understanding of the relationship between dose and image quality, and perform the examination under irradiation conditions suitable for the system and examination procedure. For example, fluoroscopy and imaging using high tube voltages generally leads to reduction of skin-absorbed doses due to its favorable X-ray penetration, although contrast may decrease slightly due to increased Compton scattering.

4. For fluoroscopy, use the minimum possible pulse rate acceptable to the operator.

5. Use a supplementary filter.

6. Distance the X-ray tube from the patient as much as possible.

7. Bring the image intensifier into a position as close to the patient as possible (avoid frequent use of geometric magnification).

8. Minimize the use of magnification during fluoroscopy and imaging.

9. For patients of small body size and in procedures in which the image intensifier remains away from the patient, remove the grid.

10. Be sure that the irradiation field is always kept in the narrowest range possible. capacity of X-ray tube systems allowing fluoroscopy even with both arms located in the irradiation field.

Figure 14 shows the positional relationship between the irradiation field and the patient's arm as it enters, and corresponding dose ratios. Even if an arm is positioned in the vicinity of the irradiation field and the presence of the arm does not affect X-ray irradiation conditions because the arm itself will not be exposed, the distance between the X-ray source and skin is decreased by the thickness of the arm, and the dose at the skin entrance surface increases. Furthermore, if an arm is located at the center of the image intensifier, more intense irradiation is required due to the presence of the arm, and the distance between the X-ray source and skin decreases, so the dose received by the part of the arm in the irradiation field is extremely high.

In irradiation with an upper arm in the irradiation field, the distance the X-ray beam travels across the patient to reach the image intensifier increases, but the intensity of radiation is exponentially attenuated within the patient's body. For this reason, lateral irradiation with an upper arm in the irradiation field results in delivery of great energy to the skin of the upper arm at the site of entrance, which the operator needs to be aware of. Every effort should thus be made to keep the upper arm away from the irradiation field to the maximum extent possible by, for example, changing the X-ray entrance angle and positioning the upper arm distal from the trunk appropriately so that it does not enter the irradiation field. ${ }^{5,17}$ 


\section{Efforts Toward Reduction of Patient Exposure Dose}

\section{A General Rules for Reduction of Exposure Dose}

Q21: Please show how to reduce the patient dose during $\mathrm{PCl}$.

A: The general rules for protection from sources of radiation outside the patient's body concern (1) time (shorten the time of irradiation), (2) shielding (block radiation), and (3) distance (ensure sufficient distance from the source). Table 2 shows specific ways of following these rules..$^{1,4,18}$

\section{B Exposure During Emergency Examination/Treatment}

Q22: A patient with acute myocardial infarction is undergoing PCl. The monitored dose has exceeded the level that can cause skin injuries, but the examination cannot be terminated prematurely because no other means of treatment is available. Is there any way of continuing the examination without causing skin injury?

A: Although priority should be placed on saving the patient's life, rather than avoiding skin injury, skin injury should be minimized to the extent possible. To avoid skin injury, the system's arm may be rotated to change the site of irradiation on the patient's skin surface to a different position. In some types of imaging systems, overlapping exposure of regions of skin to primary X-ray beams can be eliminated by rotating the X-ray entrance angle by $40^{\circ}$ or more (Figure 15); the same applies not only to the axial direction (RAO-LAO) but also the cranio-caudal direction. Since PCI is performed at an angle that enables the best identification of the lesion, it is sometimes difficult to change the angle midway, though this may need to be considered, and it is important that the operator be aware of the angles of radiation overlap of the system used in his or her institution. ${ }^{9}$

\section{Patient Variables Affecting the Onset of Radiation-Induced Skin Injuries}

Q23: Are some patients more susceptible to radiationinduced skin injury than others (due to the age and body type of the person exposed, which part of the skin is exposed, underlying disease, drugs, and other variables)? Are there any age-related differences in the likelihood of radiation-induced skin injury? If the elderly are particularly likely to suffer skin injury, is it better to consider the patient's age in deciding whether to perform restudy CAG?

A: The likelihood of radiation-induced skin injuries depends on the following variables on the patient side.

1. Age:

The biological effects of radiation are thought to vary depending on the patient's age at the time of exposure. Generally, younger cells are more sensitive to radiation. Radiation injuries are essentially the result of impairment of cell proliferation by X-rays; cells with shorter cycles of proliferation are more radiosensitive than slowly proliferating cells and resting cells. On the other hand, the rate and degree of recovery of damaged tissues also affect the manifestation of radiationinduced skin injuries. It thus remains unclear whether the elderly are particularly likely to suffer skin injuries.

2. Body type:

For patients with thick thoracic cage due to obesity, muscularity, or other factors, greater doses are required than for thin patients because of the decrease in X-ray permeability.

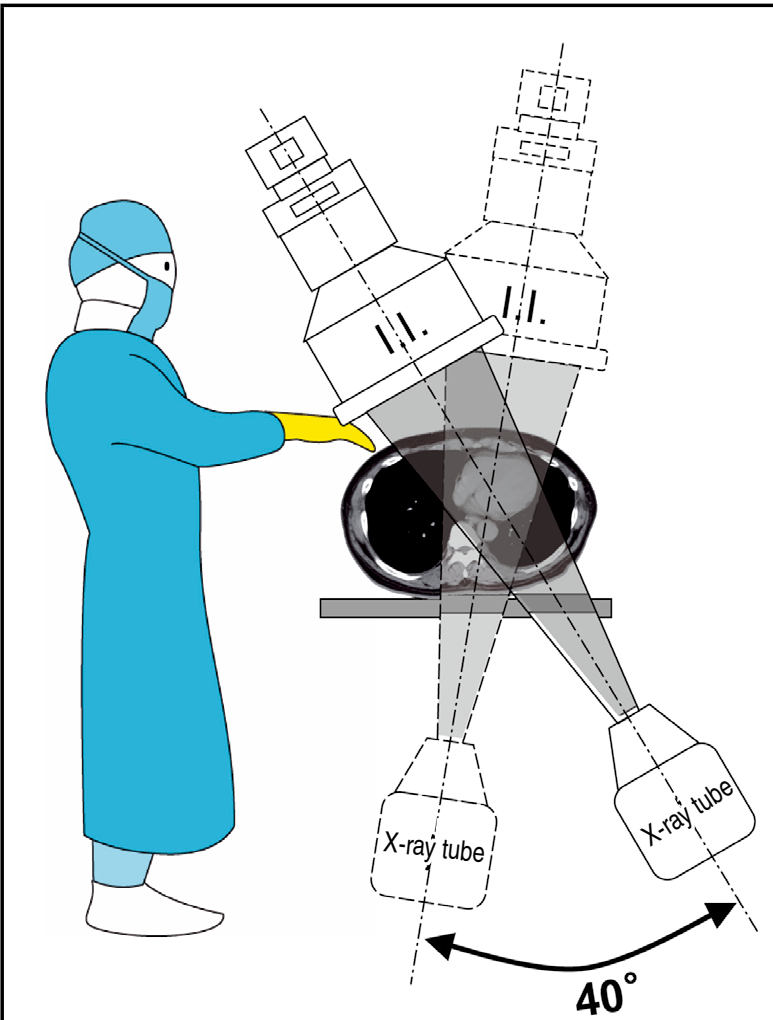

Figure 15. Example of reduction of patient skin entrance dose. When the X-ray entrance angle is changed by $40^{\circ}$, there is no overlap of exposed skin areas. I.I., image intensifier.

Additionally, in obese patients, the position of the diaphragm is relatively high, so the abdominal organs are often included in the irradiation field; the $\mathrm{X}$-irradiation dose is thus further increased. For these reasons, obese patients tend to be exposed to greater doses, and are hence more likely to suffer radiation-induced skin injury (see Q12 and Figure 6).

3. Site:

The degree of susceptibility to radiation also depends on which part of the skin receives exposure. According to Kalz et al the parts of the skin most likely to exhibit acute responses are the frontal surface of the neck and the bending portions of the limbs, such as the anterior cubital region and popliteal fossa, followed by the chest, abdomen, face, back, outer aspects of the limbs, nape of the neck, scalp, hands, and ankles. The hair follicles are more sensitive than other skin structures. 4. Underlying disease:

It is reported that radiation-induced skin injury is exacerbated in systemic sclerosis, systemic lupus erythematosus (SLE) and mixed connective tissue disease (MCTD) patients, but no clear correlation has been established. Diabetes mellitus and hyperthyroidism intensify radiation injuries. A report is available on a patient complicated by MCTD and diabetes mellitus who suffered severe necrotic ulceration after undergoing IVR. Ataxia telangiectasia patients with a homozygous genotype are particularly susceptible to radiation.

\section{Effects of drugs:}

Skin radiosensitivity also increases in the presence of chemotherapeutics such as actinomycin D, adriamycin, bleomycin, fluorouracil (5-FU), and methotrexate (MTX). It has been reported that even several months after healing of initial reactions following $\mathrm{X}$-irradiation, skin injuries recurred locally 


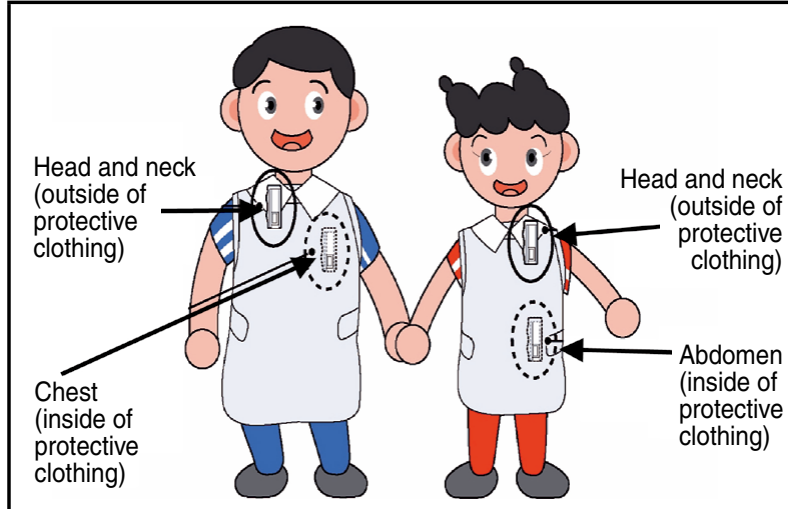

Figure 16. Positions for wearing of personal dosimeters.

only with administration of actinomycin D for several weeks.

When the clinical benefits of catheter techniques are considered very significant and outweigh the risk of radiationinduced skin injury, catheterization for the examination and/ or treatment of the target disease should be considered reasonable. It should be noted, however, that obtaining informed consent concerning the risk of radiation-induced skin injury is essential..$^{5,17}$

\section{Strategies for Reducing Exposure in Medical Personnel}

\section{A Medical Personnel Exposure}

Q24: I've heard that regarding operator exposure, the radiation produced from the patient's body is more important than that from the X-ray tube. What is meant by this? A: Since operator exposure is for the most part due to scattering of $\mathrm{X}$-rays from the patient, to reduce the dose received by the operator, emphasis should be placed on controlling the X-rays scattered from the patient. In particular, minimizing the dose received by the patient leads to a reduction in the dose received by the operator.

Q25: Persons engaged in $\mathrm{PCl}$ procedures receive high exposure doses, and I am anxious about the radiation injuries that may result from this. Please describe the radiation injuries that can occur in $\mathrm{PCl}$ personnel. How long per year can we be involved in $\mathrm{PCl}$ or $\mathrm{CAG}$ without injury to our health?

A: In PCI, since X-ray fluoroscopy is performed at high dose rates for prolonged periods of time, the exposure dose for the PCI operator, who works in the vicinity of the patient, is higher than that for other persons involved in interventional radiological procedures. Cataract can occur from exposure to a total of $2 \mathrm{~Gy}$ or more over a short period of time, or from an exposure to a total of $5.5 \mathrm{~Gy}$ or more over 3 months or longer. Accordingly, the upper dose limit for PCI personnel has been set at $150 \mathrm{mGy} /$ year for the ocular lens, and $500 \mathrm{mGy} / \mathrm{year}$ for the skin. Prevention and management of exposure should be emphasized to ensure that these upper limits will never been exceeded. Recently, it was reported that an American physician in charge of IVR developed ocular cataract even with radiation exposure that did not exceed 2 Gy. "The Chernobyl Cataract Study" has suggested that radiation cataract can occur even at relatively low doses of approximately $250 \mathrm{mSv}$; this finding is consistent with the results of studies of atomic bomb victims, astronauts, and patients undergoing X-ray computed tomography (CT) scan examination of the head. With these considerations in mind, the catheterization laboratory manager should endeavor to prevent radiation injuries in the medical personnel working in the laboratory.

Laws and regulations stipulate that the catheterization laboratory manager must ensure that persons involved in interventional radiological procedures in cardiac catheterization examinations and other interventional techniques will not be exposed to doses exceeding the following limits:

1. $100 \mathrm{mSv}$ in 5 years

2. $50 \mathrm{mSv}$ in 1 year

3. For females, $\mathbf{5} \mathrm{mSv}$ in 3 months, in addition to the two limitations above

4. For pregnant women, in addition to the above, $1 \mathrm{mSv}$ of internal exposure during the period from the time when the hospital or clinic manager becomes aware of her pregnancy as a result of her reporting it or other means to delivery

5. For the abdominal surface of a pregnant woman, $2 \mathrm{mSv}$ during the period specified in 4 . above

6. For the ocular lens, $150 \mathrm{mSv}$ in 1 year

7. For the skin, $500 \mathrm{mSv}$ in 1 year

The catheterization laboratory manager is required to ensure that the persons involved in radiological medical procedures, including CAG, carefully observe these dose limitations, no matter how many examinations they perform. To this end, it is important that efforts be made to reduce exposure by making the best use of protective clothing and protective devices, and that workers wear personal dosimeters such as glass badges and do their work in an environment controlled to avoid exposure exceeding the dose limit. ${ }^{12,19,20}$

\section{B How to Use Personal Dosimeters}

Q26: Personal dosimeters (photoluminescent glass dosimeters and optically stimulated luminescent [OSL] dosimeters) are available to wear on the head and chest. Where should we put them on? And if we put them on under protective clothing, where should they optimally be placed?

A: Figure 16 shows recommended positions for wearing personal dosimeters. During PCI procedures, two dosimeters should be worn, one inside the protective clothing and the other outside of it. For protection inside the protective clothing, personal dosimeters should be worn on the abdomen in the case of female workers, and on the chest in the case of males and women deemed without the potential to become pregnant. Outside of the protective clothing, personal dosimeters should be worn on the head and neck to monitor lens exposure doses.

\section{Types and Effects of Protective Clothing and Effects of Lead Equivalent}

Q27: What type of protective clothing should I wear during $\mathrm{PCl}$ ? Protectors with lead equivalents of $0.25 \mathrm{mmPb}$ and $0.35 \mathrm{mmPb}$ are available. Please tell me the efficiency of protection for each type of clothing compared with exposure without protective clothing. Is a $0.35 \mathrm{mmPb}$ protector more effective? Otherwise, is a $0.25 \mathrm{mmPb}$ protector sufficient?

A: Regarding protective clothing, the higher the protective performance is, the better, though generally items with high 



Figure 17. Shielding effect of protective clothing. Comparison of $0.25 \mathrm{mmPb}$ and $0.35 \mathrm{mmPb}$ (tube voltage: $120 \mathrm{kV}$ ). Comparative data with 1 dose unit received by the operator without protective clothing. I.I., image intensifier.

protective capacity are heavy. Wearing heavy protective clothing can affect the operator's concentration, and can cause lumbago.

The current version of the Japanese Industrial Standards (JIS) specifies lead-containing sheets with lead equivalents of $0.25 \mathrm{mmPb}, 0.35 \mathrm{mmPb}$, and $0.50 \mathrm{mmPb}$ as materials for protective clothing. Generally, the greater the lead equivalent is, the higher the protective performance is, though protective clothing becomes proportionally heavier with the increase in lead equivalent. Figure 17 shows the relationship between the shielding material thickness and shielding power of protective clothing between $0.25 \mathrm{mmPb}$ and $0.35 \mathrm{mmPb}$ lead equivalents. Figure 18 shows actually measured shielding effects in coronary examination. Sufficient shielding power was obtained with the $0.25 \mathrm{mmPb}$ lead equivalent, with no significant difference found in this respect between $0.25 \mathrm{mmPb}$ and $0.35 \mathrm{mmPb}$. It is recommended that the operator use a relatively light item of about $0.25 \mathrm{mmPb}$ lead equivalent in combination with other protective devices, rather than covering his or her entire body surface with heavy material. ${ }^{12,20,21}$

Q28: There are various types of protective clothings, including the apron type, which does not include lead in the back; the coat type, which includes lead both in the front and back; and the top-bottom separation type. Are there any differences among them in degree of protection from radiation exposure? Which is the ideal type?

A: Generally, wearing of protective clothing on the operator's back prevents entry of the X-rays produced when the operator is working with his or her back to the patient in flu-

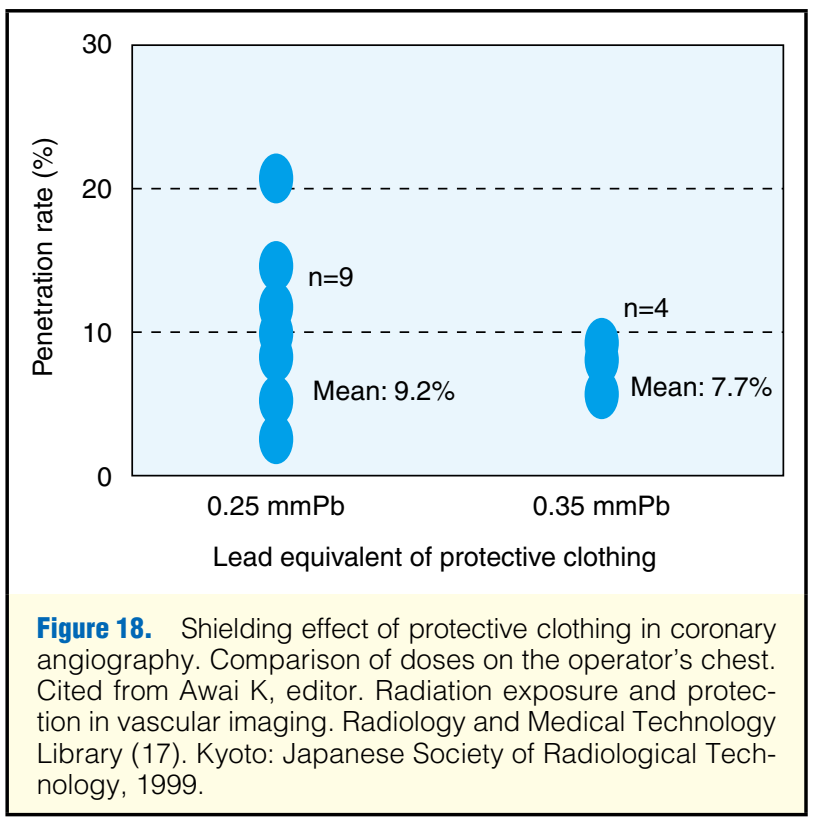

oroscopy, and entry of the X-rays scattered against the patient and further scattered against walls and equipment. When the back is covered with $0.25 \mathrm{mmPb}$ protective clothing, the tissue-absorbed dose on the back can be reduced by half or so. However, provided that the operator does not have his or her back to the patient, the amount of X-rays entering the operator's body from the back is not much, so 
the effective dose remains almost unchanged. It is thus practical for the operator to wear protective clothing of the apron type, rather than covering his or her body with protective clothing of the coat type, which is heavy and may affect operator performance, and to be careful not to have his or her back to the patient during the operation. Whereas the apron type places weight mainly on the operator's shoulders, the weight of the separate type is dispersed over the shoulders and hips, with less induction of fatigue and improved work performance. ${ }^{12,19,20,22}$

\section{Maintenance and Management of Protective Clothing}

Q29: I've heard that protective clothing is not damageresistant. Please explain quality control for protective clothing.

A: Protective clothing is comprised of a sheet-like base material uniformly containing an element of high atomic number such as lead, and a sheet of rubber or synthetic resin covering the base. Since it is tough enough that breakage

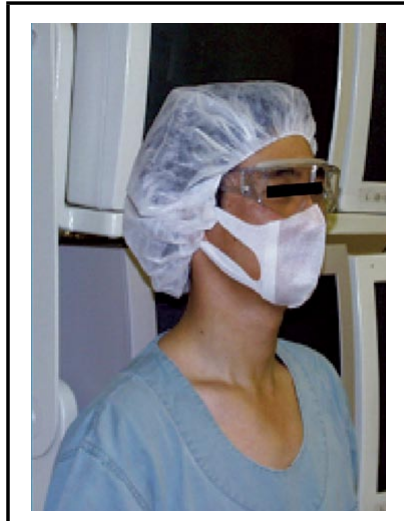

Protective spectacles: goggle type $(0.07 \mathrm{mmPb})$

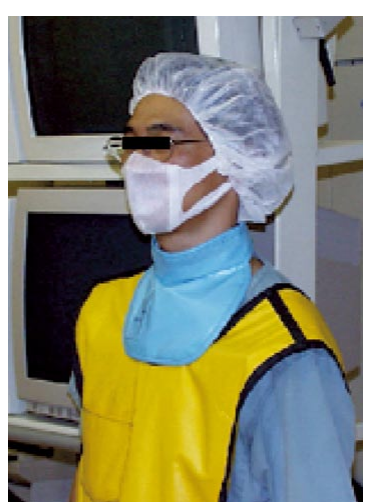

Neck guard
Figure 19. Protective devices. Cited from The guidelines on prevention of radiation-related skin disorders following interventional radiological procedures-Q\&A and Discussions. Booklet Series 3. Japan Association on Radiological Protection in Medicine; 2004. and tears do not occur readily, it might be thought that it can be used on a semi-permanent basis. However, wearing it can cause physical fatigue in the material, which in turn can produce ruptures of the shielding material inside. Adhesion of liquids such as sweat, blood, and contrast media can decrease durability. Usually, commercially available protective clothing bears an expiration date; it is necessary to stop using the clothing before that date, and to periodically implement quality control to confirm the safety of protective clothing.

In using protective clothing, the following points of note should be borne in mind.

1. Protective clothing does not completely block X-rays.

2. If protective clothing is used beyond the expiration date specified by the manufacturer, safety should be confirmed by the user's facility.

3. When storing protective clothing, avoid folding and use hangers or hooks capable of keeping it smooth.

4. Do not place excessive stress on protective clothing (do not leave it on chairs and do not sit on it).

5. Wipe off blood and contrast media adhering to protective clothing with lukewarm water or other appropriate detergents to keep it clean.

6. Check the appearance of protective clothing periodically to confirm the absence of breakage of the cover sheet.

7. It is also recommended that protective clothing be checked by fluoroscopy periodically to confirm the absence of breakage and loss of protective material..$^{20,23,24}$

\section{E Effects of Goggles and Neck Protectors}

Q30: I perform PCI many times everyday, and am curious about my exposure. Please show protective devices, other than protective clothing, that are effective during $\mathrm{PCl}$. If I wear such devices, to what extent will exposure dose be reduced?

A: Neck guards for protection of the neck and thyroid gland, protective spectacles and goggles for protection of the eyes, face guards for the face, and protective gloves for the hands and similar types of protective equipment are available. Some examples are shown in Figure 19. In choosing these devices, as with protective clothing, avoid those that are too heavy; it is recommended that you choose ones that do not prove bothersome even when worn for a long period of time. Generally, the angiographic system has the X-ray tube posi-

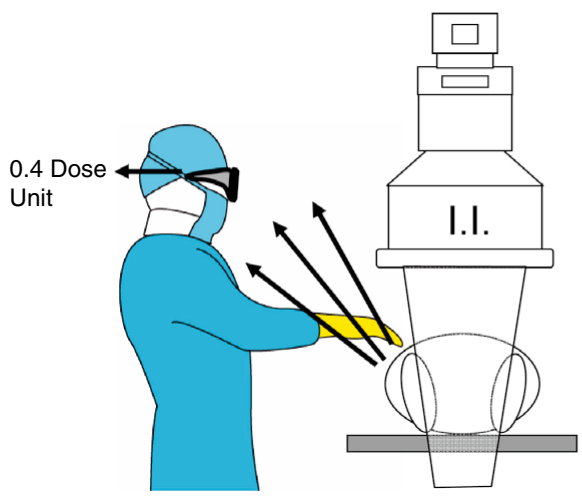

Protective spectacles $(0.07 \mathrm{mmPb})$

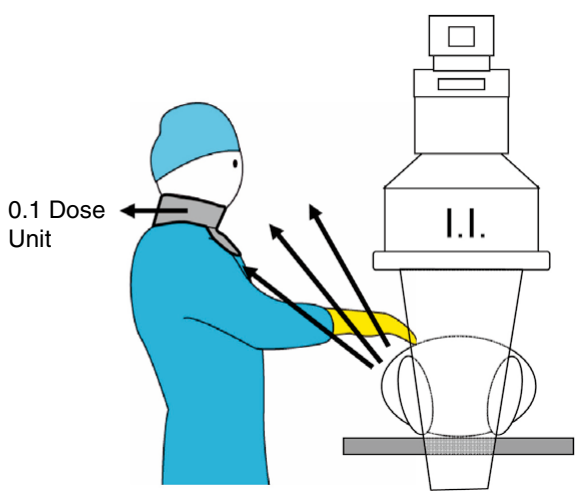

Neck guard $(0.25 \mathrm{mmPb})$

Figure 20. Effects of protective devices. Comparative data with 1 dose unit received by the operator without each protective device. I.I., image intensifier. 
tioned below the patient (under-the-table type), suggesting that the dose to the upper half of the body is not high. In PCI, however, X-rays are delivered over a broad range of directions, so protection of the upper half of the body as well is often required.

Protective spectacles are a protective device to be worn over the face to protect the face, and particularly the ocular lenses, from radiation exposure, and are made of lead-containing glass or lead-containing acrylic resin. Lead-containing glass permits use with high lead equivalent values, so it can be processed into protective devices that are strongly protective, though they are heavy. Lead-containing acrylic resin is light and highly workable, so it can be fabricated into a broad range of shapes. However, its transparency is less than that of glass, so it is difficult to fabricate with high lead equivalent values. Use of protective spectacles is also recommended not only to guard against X-ray irradiation but also to protect the eyes against scattered blood and other body fluids.

Neck guards are protective devices for protection of the thyroid gland, and are made of lead-containing sheets, as with protective clothing.

Figure 20 shows the protective effects of $0.07 \mathrm{mmPb}$ protective spectacles made of lead-containing acrylic resin and $0.25 \mathrm{mmPb}$ neck guards made of lead-containing sheets. Even relatively thin protective spectacles of $0.07 \mathrm{mmPb}$ lead equivalent have a protective effect of about $60 \%$. The $0.25 \mathrm{mmPb}$ neck guards made of lead-containing sheets have a protective effect of about $90 \%$, as with protective clothing. ${ }^{12,19,20}$

\section{F Protective Devices Recommended for Installation \\ in the Imaging Laboratory and How to Use Them}

Q31: Please describe the variety of protective devices

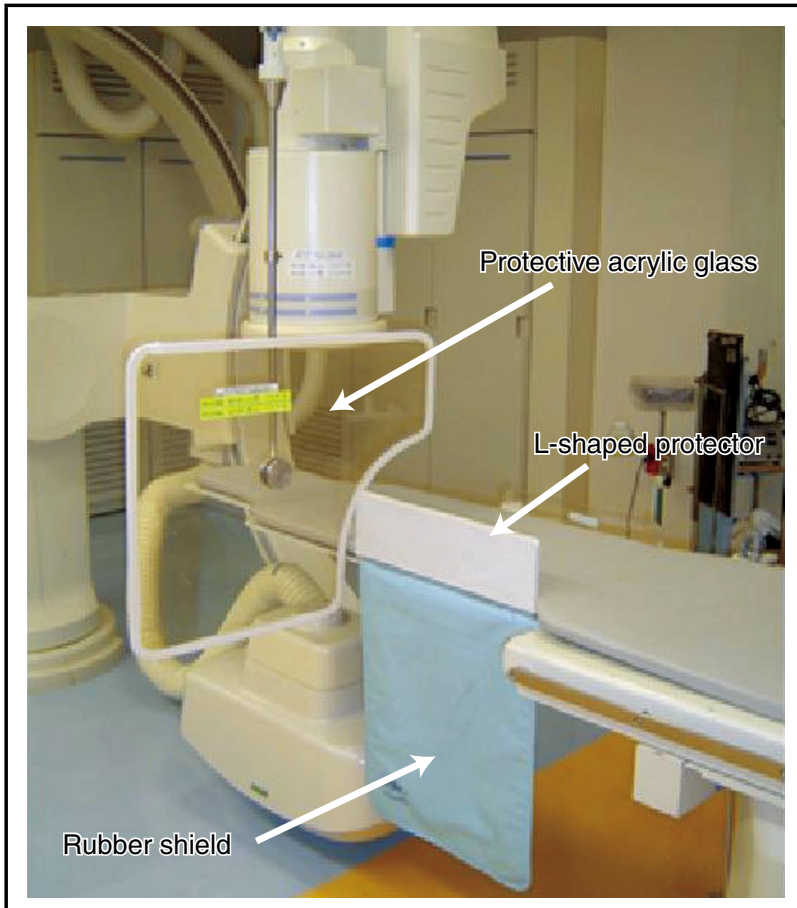

Figure 21. Protective devices attached to the instrument.

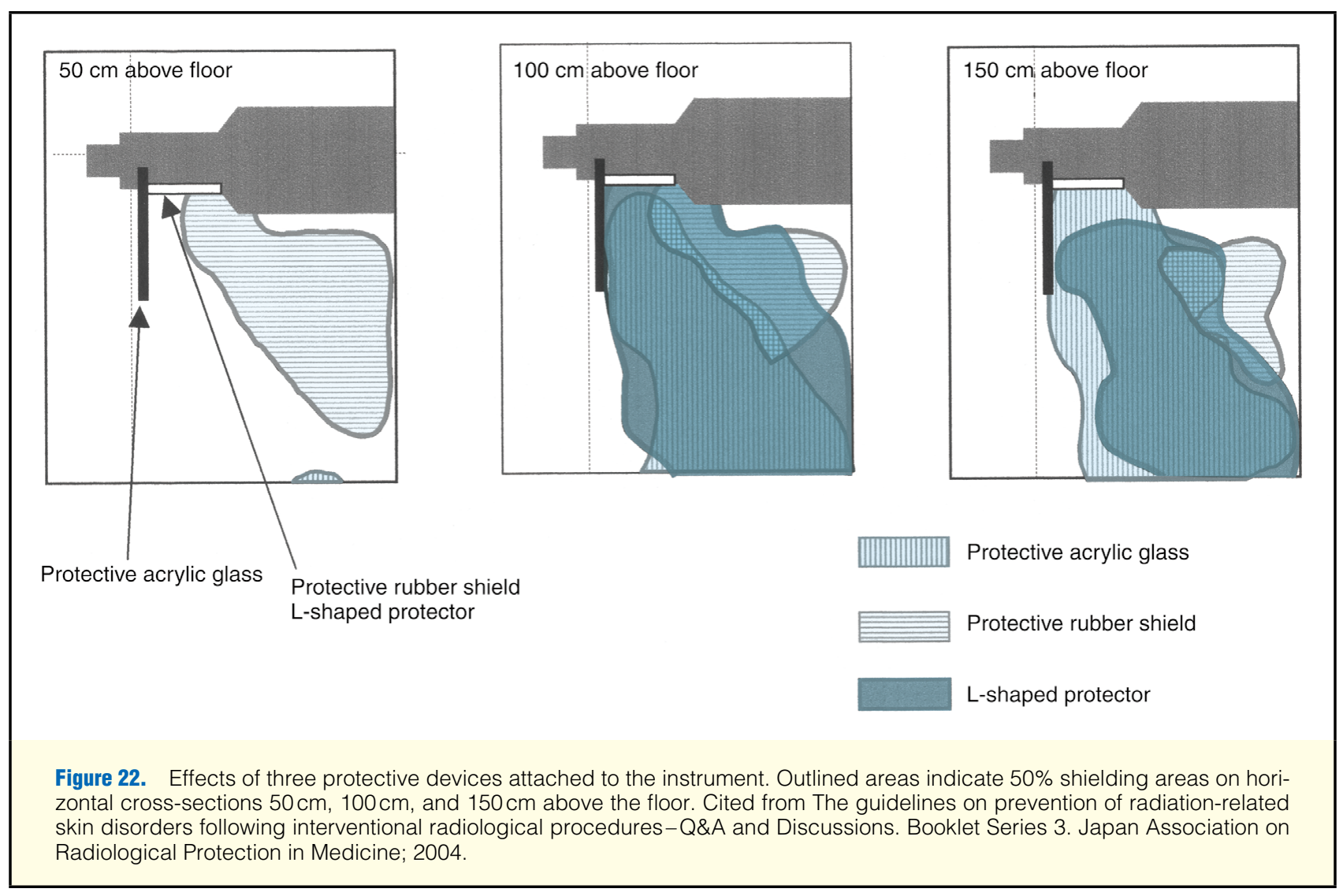




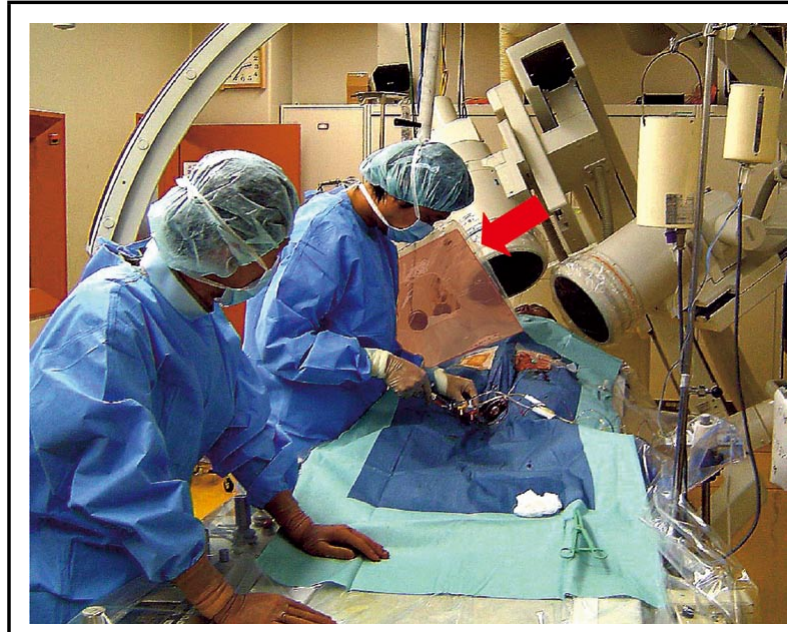

Figure 23. Effective position of guard screen (arrow).

that are helpful in the catheterization laboratory where $\mathrm{PCl}$ is performed, and show where to set them for effective use and how to use them.

A: When a protective device is attached to the imaging system, medical personnel can lessen their fatigue since wearing lightweight protective clothing is sufficient to obtain the desired protection. If a single protective device is used to obtain all protection, the increased overall size hampers movement of the system's arm and catheter table, so it is recommended that a number of protective devices of various shapes be combined as appropriate for the patient's position. Protective devices recommended to be installed in the catheterization laboratory room are shown below. Their appearances and shielding effects are shown in Figure 21 and 22. It can be seen that combining the three types produces a still broader range of shielding effect.

1. Type for protection of the lower half of the operator's body (rubber shield)

These are made of lead-containing rubber, to be suspended from the catheter table.

2. Type for protection of the operator's abdomen (L-shaped protector)

L-shaped protective devices for insertion between the catheter table and the patient's back are both radioprotective and function as a patient arm rest. Although the protective effect increases with the height of the screen portion, workability decreases. The device shown in Figures 21, 22 is $15 \mathrm{~cm}$ high.

3. Type for protection of the upper half of the operator's body (protective acrylic glass)

Lead-containing acrylic panels attached directly to ceil-
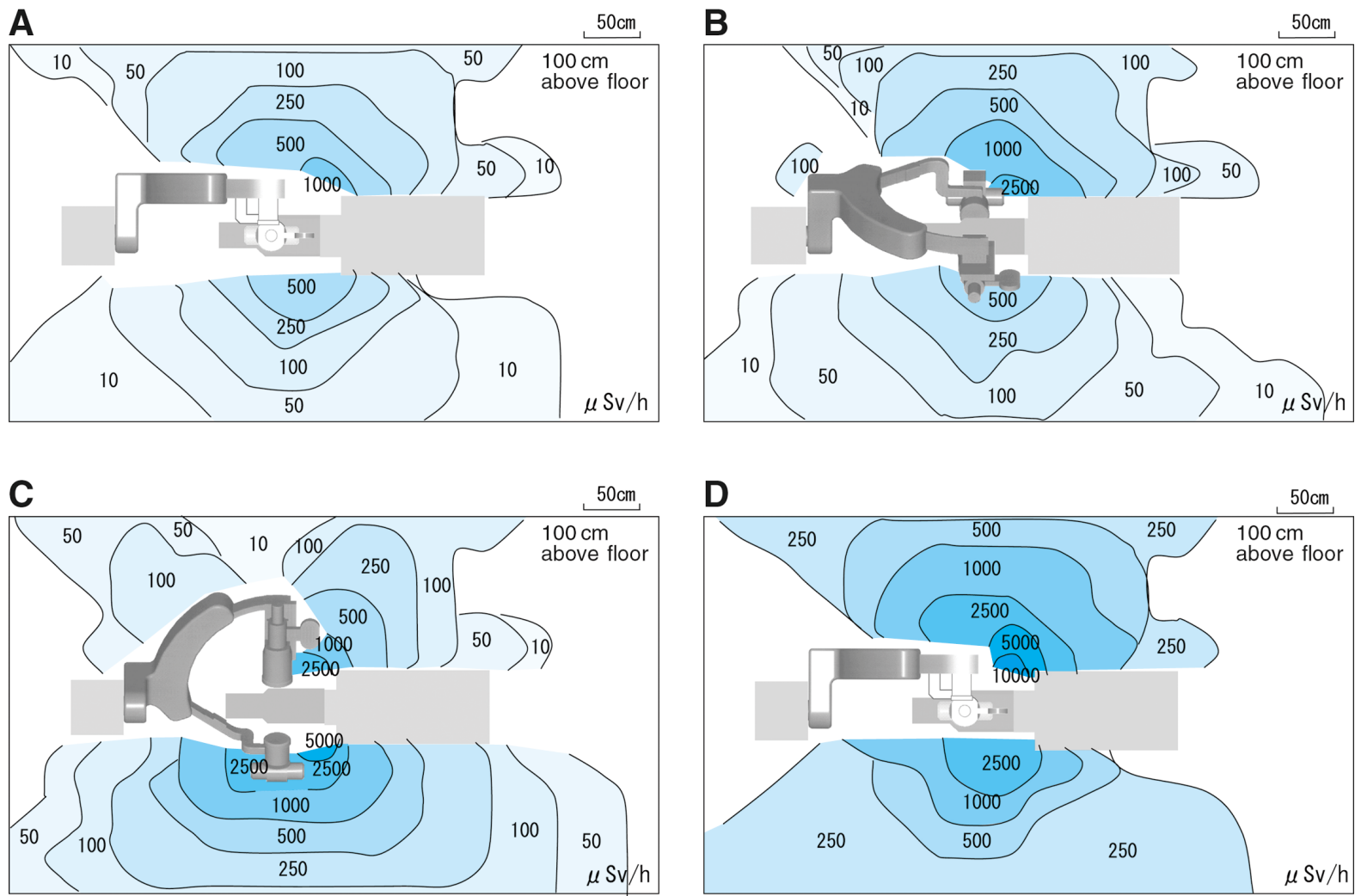

Figure 24. Dose distribution in the catheterization laboratory. (A) Fluoroscopy in posterior-anterior (P-A) view. (B) Fluoroscopy in right anterior oblique (RAO) $30^{\circ}$ view. (C) Fluoroscopy in left anterior oblique (LAO) $60^{\circ}$ view. (D) Imaging in P-A view. Cited from Awai K, editor. Radiation exposure and protection in vascular imaging. Radiology and Medical Technology Library (17). Kyoto: Japanese Society of Radiological Technology, 1999. 
ings or placed on ceiling rails are commonly used. If a panel of this type is placed on a ceiling rail, a broad range of motion is obtained; however, since it can interfere with the image intensifier and other equipment at some angles of the system's arm, caution is needed in handling the panel. X-ray exposure to the operator comes for the most part from secondary X-rays scattered against the patient. For this reason, the protective panel is effective if placed between the patient and the operator (not between the operator and the X-ray tube), and as close to the patient as possible. Figure 23 shows an example of placement for maximum effect. ${ }^{12,19,20}$

\section{G Dose Distribution in the Catheterization Laboratory}

Q32: Please show the dose distribution in the catheterization laboratory during $\mathrm{PCl}$.

A: Since knowledge of the dose distribution in the catheterization laboratory allows smooth operation with lower exposure doses, it is important that medical personnel entering the laboratory be aware of this distribution.

\section{Dose distribution during fluoroscopy}

Figure 24A, 24B, and 24C show the dose distributions for various directions of entry of X-rays. In PCI, X-rays are projected from different directions, and the dose distribution changes with each change in direction; however, in general, greater doses are delivered on the side where the X-ray tube is present. Hence, the dose is greater on the patient's left side in the RAO direction, and on the patient's right side in the LAO direction and left lateral direction.

\section{Dose distribution during imaging}

The dose rate is more than 10 times greater during imaging than during fluoroscopy (Figure 24D). Even in the peripheral areas of the catheterization laboratory, where the dose rate is relatively low during fluoroscopy, the dose rate occurring during imaging is comparable to that observed at the operator's position during fluoroscopy. It is recommended that medical personnel working in the catheterization laboratory use a guard screen during the imaging procedure, or leave the laboratory.

Taking into account these dose distributions, ICRP Publ.85 (Avoidance of Radiation Injuries from Medical Interventional Procedures) recommends that the operator stand on the image intensifier side. However, this is often impossible or unrealistic, depending on the type of examination and the shape of the system. It is feasible for the operator to stand on the opposite side of the image intensifier with appropriate protective devices. ${ }^{12,21}$

\section{H Pregnancy of Female Medical Personnel}

Q33: I am a female physician who performs angiography, and have become pregnant. What actions should I take?

A: First, you need to inform the facility manager of your pregnancy. The manager must ensure that fetal exposure does not exceed the dose limit. Laws and regulations stipulate that the upper limit of equivalent dose on the abdominal surface during gestation, between the time of establishment of the diagnosis of pregnancy and delivery, is $\mathbf{2} \mathbf{m S v}$. It is also legally required that records be kept on the equivalent dose on the abdominal surface of the mother every month and the cumulative dose over the gestational period. In the case of fetuses, public dose limits apply, so the cumulative absorbed dose received by the fetus during the period from fertilization to delivery should not exceed $1 \mathrm{mSv}$. However, although laws and regulations indicate that the exposure status of pregnant female medical staff must be adequately managed, they do not require pregnant women to completely avoid work involving the use of radiation or radioactive substances, nor do they prohibit them from accessing, or working in, specified areas with radiation exposure. It is important that the persons concerned are always aware of these facts while at work.

It is recommended that the manager keep available for female workers a form for notification of pregnancy to the manager or equivalent individual, and obtain information on each pregnant worker so as to secure a good work environment for them. In all cases, upon learning of the pregnancy of a female medical staff member, the manager must consult with her closely to ensure that she will be able to do her work comfortably while she is pregnant.

\section{Education and Re-Education of Medical Personnel}

Q34: What are the legal requirements concerning education and re-education related to radiation for physicians, nurses, and radiologic technologists engaged in $\mathrm{PCl}$ ?

A: According to the Laws Concerning the Prevention from Radiation Hazards due to Radioisotopes and Others, Ministry of Health, Labour and Welfare (MHLW) Ordinance on Prevention of Ionizing Radiation Hazards, the Rules of the National Personnel Authority, and similar laws and legulations, education and training of medical personnel in radiology is mandatory. In addition to the mandatory education/ training in radiology, individuals who are engaged in duties involving the delivery of radiation to patients must educate themselves to obtain knowledge and expertise proactively to ensure their own and their patients' safety. The MHLW Ordinance stipulates that special education concerning radiation safety be provided for persons engaged in operation of $\mathrm{X}$-ray systems.

\section{[Items for education and training]}

1. Effects of radiation on the human body [30 minutes]

2. Safe handling of radioisotopes and radiation generators [4 hours]

3. Laws and regulations concerning the prevention of radiation injuries due to radioisotopes and radiation generators [1 hour]

4. Rules on prevention of radiation injuries [30 minutes]

Note that for those with adequate knowledge and skills, all or part of the education/training programs may be skipped.

\section{[Frequency]}

The Laws Concerning the Prevention from Radiation Hazards due to Radioisotopes and Others indicates that healthcare professionals in radiology should be educated and trained once before their first access to controlled areas, and every period that does not exceed 1 year after such access.

\section{J Requirements for Labor Accident Indemnification Approval}

Q35: Please show the requirements for labor accident indemnification approval in case of the onset of cancer in a healthcare professional in radiology.

A: If a person engaged in radiological practice develops a radiation injury due to radiation received during radiationrelated work, he or she can receive compensation for medical care and the like based on the Workers' Accident Compensation Insurance Act (for national public officers, the National Public Officers' Accident Compensation Act applies). Regarding the requirements for approval, criteria are available for 
acute radiation syndrome, acute radiation-induced skin injuries, chronic radiation-induced skin injuries, radiation-induced hematopoietic disorders, leukemia, and cataract. For example, one of the requirements for approval for leukemia is an onset of illness later than 1 year after exposure, with confirmation of the fact that the sufferer received a cumulative dose of $5 \mathrm{mSv}$ or more per year.

\section{Management of Imaging Systems}

\section{A Maintenance and Management of Imaging Systems}

Q36: Please show the points to note for users of angiographic systems in maintaining the quality of such systems. A: The amount of X-rays that a patient is exposed to cannot be kept constant unless the tube voltage and tube current are constant. Variation in X-ray quality influences the onset of skin injury in patients, and the size of the irradiation field is closely related to both patient and operator exposure doses. In ensuring the safety of angiographic systems and maintaining good quality and performance, it is important that not only self-inspections but also periodic maintenance and inspections be implemented by the manufacturers of the systems. In particular, the brilliancy of image intensifiers decreases over time, so if reduced brilliancy is left as is, the automatic exposure mechanism will adjust the dose, resulting in delivery of a greater exposure dose to the patient. Although dose variation among different institutions may be due, in part, to intrinsic features of the systems used, the status of implementation of dose adjustment during system inspections may also affect it. It is important that on the occasion of periodic inspections, the brilliancy of the image intensifier be measured, and that the iris diaphragm and other components affecting the quality of images be adjusted whenever necessary to prevent the dose from increasing.

Note that if the brilliancy falls beyond the adjustable range, increase in the dose irradiated to the patient will be inevitable; replacement of the image intensifier must then be considered.

The Japan Industries Association of Radiological Systems (JIRA) has specified two cases in which the image intensifier should be replaced with a new one:

1. In cases in which the reference conditions as of the time of installation cannot be maintained even after adjustment, and the exposure dose has increased by $50 \%$ or more from the initial level.

2. In cases in which the X-ray conditions as of the time of installation can be maintained by adjustment, but the diagnostic performance has evidently decreased from the initial level to an extent that hampers diagnosis. ${ }^{24-28}$

\section{B Measures to Reduce Exposure}

Q37: I've heard that new angiographic systems are provided with a number of functions for ensuring the safe performance of $\mathrm{PCl}$. When such a system is used, don't skin injuries occur in the patient?

A: Recently developed angiographic systems suitable for PCI are provided with digitized features and many PCI aid functions as shown below. These functions, if utilized effectively, enable the operator to perform PCI smoothly while reducing both patient and operator exposures.

1. Low pulse rate fluoroscopy function is included to reduce exposure.

2. A supplementary filter to reduce exposure is provided.

3. Map image display and roadmap functions for smooth advancement of the catheter and guidewire to the target site are included.

4. Image storage and retrieval are facilitated by digital image acquisition.

5. Digital fluoroscopy is available, offering good visibility of the guidewire and stent.

It should be understood, however, that these functions never ensure the prevention of skin injuries, although they include measures to reduce exposure. Because erroneous use can lead to irradiation at higher-than-expected dose rates, it is important that the user have a clear understanding of these performance features before using them. ${ }^{9}$

\section{Non-Coronary Intervention}

\section{A Precautions in Head and Neck IVR}

Q38: Please show the points to note for head and neck IVR, such as in internal carotid artery stenting.

A: The head has hair: Which is a significant difference from the skin of the trunk irradiated during cardiac intervention. Exposure to doses exceeding $3 \mathrm{~Gy}$ can cause temporary epilation. Even when it is temporary, epilation can have a major mental impact on the patient, so you should always be sure to the extent possible to avoid irradiation of the same site in the head for a prolonged period of time. Because direct exposure of the patient's eyes to X-rays can induce cataract, shielding and irradiation angle must be taken into account. According to ICRP Publ.85, the threshold dose for cataract caused by a single exposure is $\mathbf{2} \mathbf{G y}$; this document also suggests that exposure to $5 \mathrm{~Gy}$ or more may produce progressive change. In ICRP Publ.60, it is stated that lens opacity without visual impairment can occur even with exposure of $0.2 \mathrm{~Gy}$ or less, so prolonged irradiation of the lens must to the extent possible be avoided. ${ }^{4}$

\section{B Precautions in Percutaneous Transluminal Angioplasty} (PTA) in Lower Limbs

Q39: Please show what to note during lower limb PTA.

A: PTA is an effective means of treatment for arteriosclerosis obliterans (ASO), and the number of cases in which it is performed is steadily increasing in Japan. Since patients with ischemic heart disease often develop ASO as a complication, cardiologists are now more commonly engaged in its treatment. Because of the proximity of the puncture site and the treatment site, the operator is exposed to relatively large amounts of scattered X-rays. In addition, a large-diameter image intensifier is often used, and produces larger amounts of scattered $\mathrm{X}$-rays than a small-diameter image intensifier. In performing examination for PTA, it is important that the irradiation field be narrowed to the maximum possible extent required, and that the appropriate image intensifier size be used.

Because the target vessels are often delineated in the posterior-anterior views rather than the oblique views commonly used in PCI, protective rubber shields and protective acrylic glass equipped with the radiation system or the examination room are effective (Figure 21). It is recommended that the operator obtain radioprotection using these protective devices. ${ }^{9,12,20}$ 


\section{Electrophysiological Examinations and Treatments}

A Exposure Doses to Patients Undergoing Catheter Ablation Q40: What are the distinct features of radiation exposure in electrophysiological examinations and treatments, such as catheter ablation and pacemaker implantation compared with those in $\mathrm{PCl}$ ? Are there any aspects of such procedures that require special attention as regards protective measures?

A: In electrophysiological examinations and treatments, fluoroscopy is mainly used, with imaging performed only minimally. In addition, since catheter electrodes, which are more easily recognizable than guidewires, are used, it is common practice to perform the examination at reduced fluoroscopy pulse rates. These features offer protective advantages compared with PCI. It should be noted, however, that since catheter ablation is likely to involve continuous fluoroscopy at a fixed X-ray entrance angle, irradiation of the same site is often prolonged. In case of a long period of irradiation in the LAO position, in particular, concentrated exposure can occur in the right subscapular region and right upper limb; special caution is required with regard to this. Efforts should be made to use low pulse rate fluoroscopy, and to keep the upper arms away from the irradiation field, by, for example, distancing the upper arms from the trunk as far as possible.

\section{B Precautions for Pediatric Patients}

Q41: What are the points to note for pediatric patients? I've heard that paralysis can occur when the upper limbs are placed in a raised position for a long time. Is there any effective method of preventing this?

A: When a pediatric patient undergoes catheterization or intervention under general anesthesia, frontal and lateral views using biplane fluoroscopy are often used; to secure a field for lateral fluoroscopy, the upper limbs are sometimes immobilized while raised. Prolonged immobilization can cause injuries to the brachial plexus, brachial nerve, ulnar nerve and other nerves which in turn can result in paralysis and hypesthesia in the ulnar side of the palm, as well as distal from the shoulder joints or cubital joints. This is attributed to ulnar nerve hyperextension due to elbow abduction under the weight of the arm, or to damage to the entire brachial nerve due to axillary hyperextension, as a result of inappropriate immobilization. Although signs and symptoms such as motor paralysis and hypesthesia are for the most part transient, recovery can take 6 months or longer in some cases. This can be prevented by avoiding immobilization in a constant limb position. If immobilization is unavoidable, it is recommended that the immobilization be loosened, with upper limb adduction, to achieve transient decompression of the nerves, at intervals of about 30 to 60 minutes.

\section{Nuclear Imaging}

\section{A Precautions in Performing PCI on the Same Day as Thallium (TI) Myocardial Scintigraphy or the Following Day}

Q42: Please indicate the precautions in performing cardiac catheterization on the day of or the day after TI scintigraphy to evaluate myocardial viability. What issues exist concerning the risk of radiological exposure due to isotopes remaining in the patient's blood and catheter devices?

A: Because the amounts of isotopes administered to the patient in nuclear imaging are small, it is generally believed that cardiac catheterization poses no problem related to exposure of the operator and other staff even when it is performed on a patient who received an isotope on the same or the following day. However, because the patient's blood contains a residual portion of the isotope, although it may be quite small, the injection needles, catheters, and related equipment used in the cardiac catheterization may be contaminated. For this reason, used disposable instruments should be stored until their radioactivity level becomes undetectable with a counter, and then disposed of as infectious waste. In addition, since isotopes are excreted at high levels in the urine, the patient's urine should be handled with greater care than the blood. The urinal bags and used diapers of patients undergoing nuclear imaging should be handled in the same fashion as catheters and injection needles.

\section{B Exposure of Medical Personnel Engaged in TI Myocardial Scintigraphy}

Q43: I perform intravenous injection of isotopes during TI myocardial scintigraphy. What is the extent of exposure of my fingers and body? Is any method available to reduce this exposure?

A: According to a 1995 survey by the Japanese Society of Nuclear Medicine Technology, exposure doses to the trunk of radiologic technologists involved in nuclear imaging were $\leq 0.2 \mathrm{mSv} / \mathrm{month}$ in $\geq 75 \%$ of the institutions that participated in the survey, whereas the exposure doses to the fingers exceeded $0.5 \mathrm{mSv} / \mathrm{month}$ in as many as $30 \%$ of the institutions. Many of the recently launched isotopes are supplied in shielded syringes with tungsten and lead glass. As such, greater consideration is given to reduction of exposure of medical personnel than ever, although no shielding is provided on the injection needle side and the plunger side. Actual measurements showed that the plastic material used on the plunger side had only a low shielding effect. Even when a tungsten plunger was used, the shielding effect estimated from the leakage rate was about $75 \%$ for technetium (Tc)$99 \mathrm{~m}$. In handling the shielded syringes as well, quick and appropriate performance of procedures is an effective way to reduce exposure from isotopes used for scintigraphy. ${ }^{29}$

\section{How to Handle Subcutaneous Leakage of Isotopes}

Q44: I have erroneously injected an isotope into subcutaneous tissue of a patient during myocardial scintigraphy. Please tell how to handle this.

A: Subcutaneous leakage on intravenous injection is not rare in the clinical setting. If a subcutaneously leaked isotope remains localized at the site, it is possible that the local tissue will absorb a high dose, indicating that caution is needed even if the isotope has a short half-life. In a case reported in Japan, Tl-201 leaked from subcutaneous blood vessels during myocardial scintigraphy; despite immediate treatment, the portion of the skin around the site of leakage necrotized 2 weeks later, followed by ulceration with scar epithelialization 3 months later, and severe scarring, depigmentation, skin atrophy, vascular dilation, and peri-pigmentation in the center of the affected part 4 years later; chronic radiation dermatitis was eventually diagnosed. If leakage of an isotope is detected during examination, it is important that the maximum expected skin-absorbed dose be roughly calculated by, for example, quickly obtaining images of the site of leakage to obtain dose information, and that the patient be followed over time. ${ }^{30,31}$ 



Figure 25. Comparison of patient exposure doses in coronary angiography and coronary CT. Imaging conditions: scanography $120 \mathrm{kV}, 10 \mathrm{~mA}$; positioning scan, $135 \mathrm{kV}, 300 \mathrm{~mA}$, pitch 1.0; Real Prep, $135 \mathrm{kV}, 50 \mathrm{~mA}$; contrast-enhanced helical scan, $135 \mathrm{kV}$, $450 \mathrm{~mA}$, pitch 0.19. Angiographic data courtesy of Kanazawa University. CT, computed tomography; I.I., image intensifier.

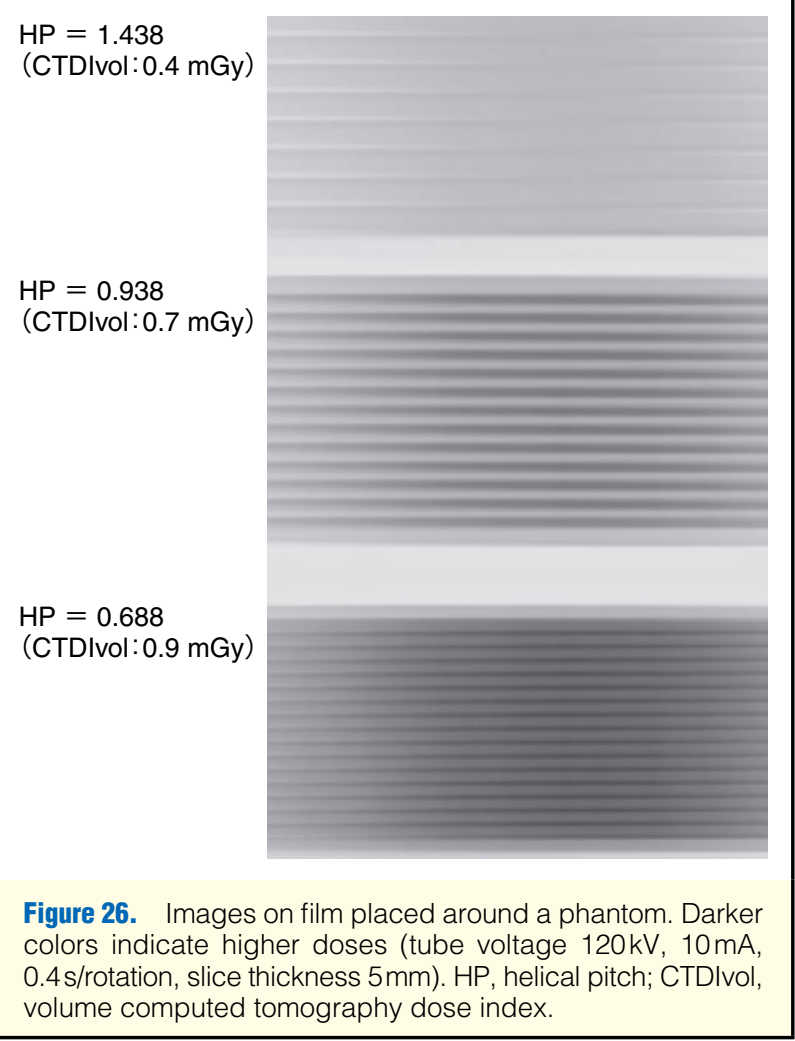

11. CT

A Exposure Dose to Patients Undergoing Coronary CT Q45: Recently, evaluation of coronary lesions by multi- detector computed tomography (MDCT) has become increasingly common. What is the average exposure dose in such cases? How about a comparison with CAG?

A: A commonly used imaging protocol for coronary CT is as follows:

1. Scanography: Obtain an approximate imaging range.

2. Scout scan for positioning: Determine the range of scan for contrast imaging.

3. Scan to determine appropriate timing of contrast imaging scan (Real Prep): After intravenous injection of contrast medium at a rate of 3 to $4 \mathrm{ml} / \mathrm{s}$, the scan is repeated at constant position. While monitoring the CT value of the region of interest (ROI) set in the ascending aorta or cardiac cavity, the timing to start contrast-enhanced helical scan is determined.

4. ECG gated, contrast-enhanced helical scan: The helical scan data are obtained with simultaneous recording of the ECG.

Doses in a phantom whose images were taken using this protocol are shown in Figure 25.

The dose received by the patient is greater in coronary CT than in ordinary chest CT. In ordinary CAG for diagnostic purposes, the maximum skin entrance dose is several hundred mGy, and coronary $\mathrm{CT}$ produces similar doses. In coronary $\mathrm{CT}$, it is necessary to choose as thin a slice thickness as possible $(0.5 \mathrm{~mm})$ to obtain high-resolution 3-demensional (3D) images, and to choose a small helical pitch (HP) to obtain images in multiple time phases. Figure 26 shows the results of observation of scan traces with three different pitches in a phantom with a film placed around it. Although smaller HPs produce more dense images, they also produce greater doses. This is the reason why the patient receives larger doses in coronary CT.

Figure 27 shows dose distributions in CT. In CAG, the highest dose occurs on the skin surface where $\mathrm{X}$-rays have 


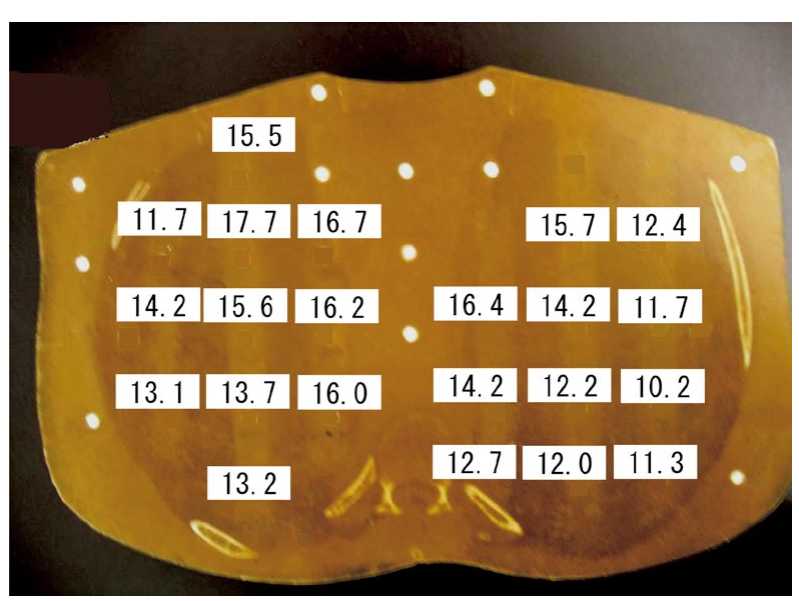

Chest CT

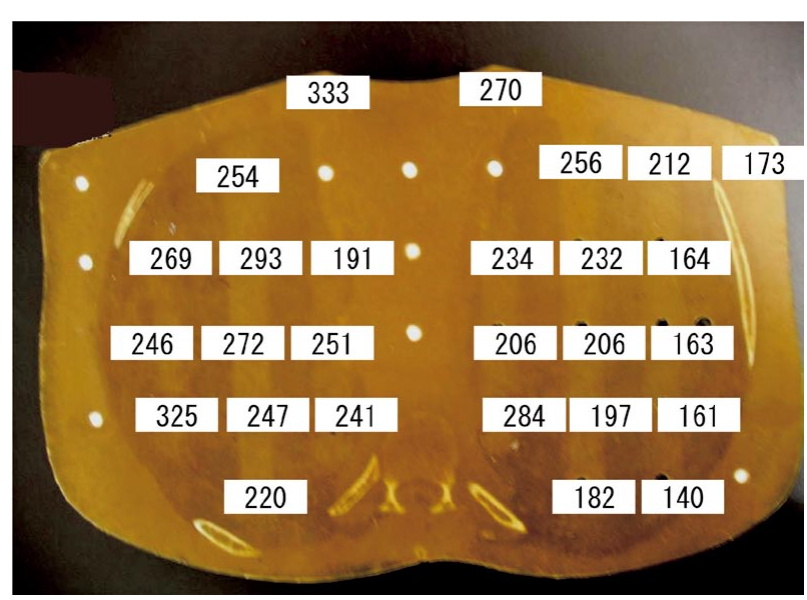

Coronary CT

Figure 27. Comparison of doses received by a patient undergoing chest CT and coronary CT. Data courtesy of Fujita Health University. CT, computed tomography.

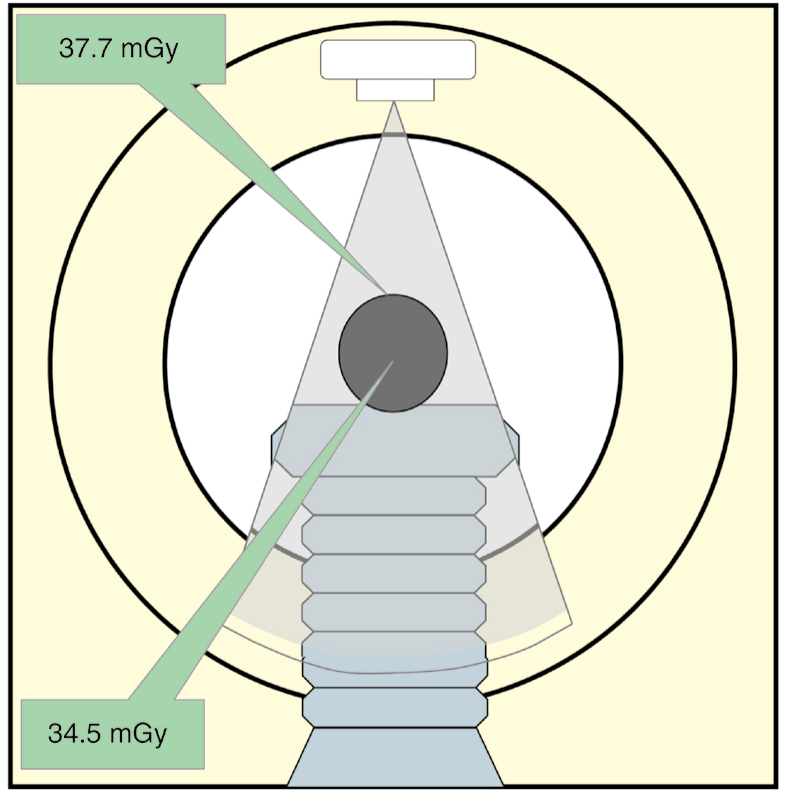

Phantom diameter: $16 \mathrm{~cm}$

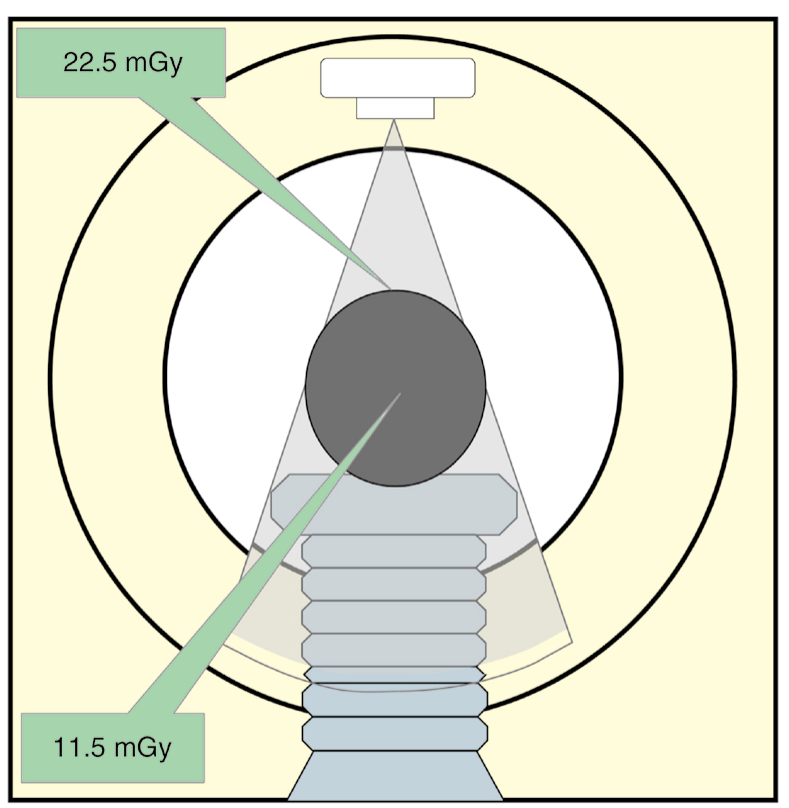

Phantom diameter: $32 \mathrm{~cm}$

Figure 28. Comparison of exposure doses for patients of different body types in coronary CT. Irradiation conditions: $120 \mathrm{kV}$, $200 \mathrm{~mA}, 1.0$ sec. CT, computed tomography.

entered. In CT, the dose in the center of the body is not widely different from the skin surface dose because the trunk is irradiated circumferentially. For this reason, with the same maximum dose, a greater effective dose is produced in CT, where similar doses are irradiated over the entire imaging area, than in CAG, where doses are localized on the skin surface. The risk of carcinogenesis thus appears to be higher with CT. However, it remains unclear whether this difference is clinically significant. ${ }^{31-34}$
Q46: Does the exposure dose vary depending on the patient's body type in CT as in CAG? Is this variation clinically problematic?

A: When CT images are taken under the same conditions, patients of smaller constitution receive greater exposure doses. Figure 28 compares doses in the centers of, and around, two phantoms $16 \mathrm{~cm}$ and $32 \mathrm{~cm}$ across, respectively, imaged under the same irradiation conditions. Because the X-rays used for diagnostic purposes only minimally permeate the 
patient's body and lose much of their energy, irradiation under the same conditions produces greater mean absorbed doses in lighter than in heavier patients. In addition, patients of smaller constitution exhibit smaller dose differences between the center and surrounding area, and hence receive higher doses at any position on the phantom; irradiation conditions suitable for the patient's body type should therefore be chosen before obtaining images. When a child is being examined, in particular, measures to prevent excessive irradiation, such as reducing the tube current, should be taken. Automatic irradiation control, a function widely used recently to allow automated adjustments of irradiation conditions according to the acquisition site based on scout views, is expected to facilitate imaging at appropriate doses without affecting image quality.

\section{B Effects of the Use of Multi-Row Detectors}

Q47: Does the patient exposure dose increase with the number of detectors in MDCT? What is the difference in exposure dose from ordinary CT in major blood vessels?

A: Basically, provided that the slice thickness and HP are the same, the dose received by the patient is the same irrespective of the number of detector arrays. Compared with ordinary CT, there is no notable difference. In MDCT, however, images are sometimes acquired with slice overlaps to obtain clear 3D images. In such cases, greater doses are absorbed in the regions where scans overlap. ${ }^{33,34}$

\section{Effects on Pacemaker and Implantable Cardioverter Defibrillator (ICD)}

Q48: What are the points to note in performing CT examination on a patient with a pacemaker or ICD?

A: It was recently reported from more than one institution that partial resets occurred during CT of patients with an implanted pacemaker or ICD. This was attributed to unwanted currents produced as a result of a photoelectric effect induced by irradiation of the CMOS circuit, which amplifies the electrical excitation of the heart. As a result, in patients with a pacemaker, transient suppression of pacing pulse output due to oversensing can lead to cardiac arrest. In ICD, the defibrillator can operate erroneously due to oversensing.

If $\mathrm{CT}$ or delivery of a relatively high dose of radiation is performed on a patient with either device implanted, attention should be paid to the following:

1. How to handle pacemaker-related problems

- Do not irradiate the implantation site for 5 seconds or longer.

- If an X-ray beam must be continuously directed at the implantation site for 5 seconds or longer, take appropriate measures to distance the irradiation site from the pacemaker as much as possible, such as asking the patient to raise both of his or her arms.

- If irradiation of this type for 5 seconds or longer cannot be avoided, perform the examination in the fixed pacing mode without competitive pacing, or prepare for external pacing in case of pacemaker-related problems.

2. How to cope with ICD-related problems

- Do not irradiate the implantation site.

- If X-ray irradiation of the implantation site is unavoidable, take appropriate measures to distance the irradiation site from the ICD as much as possible, such as asking the patient to raise both of his or her arms.

- If irradiation of this type cannot be avoided, perform the examination only after turning off the tachycardia detection function.
- Be prepared to use an temporary external defibrillator or a external pacing during the examination.

In all cases, a specialist capable of responding quickly, including reset cancellation, should attend the examination, and it is essential that the pulse be monitored via an ECG monitor. ${ }^{35}$

\section{Examination and Treatment of Pregnant Patients}

Q49: What are the points to note in catheterization studies of pregnant women in the middle gestational stage or later?

A: Irradiation of pregnant patients should be avoided to the extent possible. Since maternal health benefits the fetus, any required examination should be performed with due caution concerning the matters indicated below. In such cases, it is important that adequate informed consent be obtained from the patient after a full explanation of the possible effects on the fetus and other related matters, and that the dose received by the fetus be limited to $100 \mathrm{mGy}$ or less. When the irradiation field is in the lower abdomen (direct exposure on the fetus), or when a catheterization study of the pelvis is required, it is necessary to confirm the need for examination, and to determine whether the examination can be delayed until the end of gestation. In examinations in which the dose received by the fetus is likely to markedly exceed $100 \mathrm{mGy}$, a method of examination not involving the use of radiation should be considered.

To minimize the dose to which the fetus is exposed, the following should be noted.

1. In examination of parts of the mother's body relatively far from the fetus, such as the chest, upper limbs, and head, a catheter insertion site that does not cause direct exposure of the fetus to irradiation (cubital artery, radial artery, etc) should be chosen.

2. Rigorous use of common techniques for reduction of exposure (eg, low pulse rate fluoroscopy, limitation of the irradiation field, use of a supplementary filter, and shortening of the distance to the image intensifier).

3. Determine the dose to which the fetus is exposed. ${ }^{36}$

\section{References}

1. Hirshfeld JW Jr, Balter S, Brinker JA, Kern MJ, Klein LW, Lindsay $\mathrm{BD}$, et al. ACCF/AHA/HRS/SCAI clinical competence statement on physician knowledge to optimize patient safety and image quality in fluoroscopically guided invasive cardiovascular procedures: A report of the American College of Cardiology Foundation/American Heart Association/American College of Physicians Task Force on Clinical Competence and Training. Circulation 2005; 111: $511-$ 532.

2. Manual on management and measurement of radiation exposure 2003. Japan Association on Radiological Protection in Medicine, 2003 (in Japanese).

3. ICRP Publication 60: 1990 Recommendations of the International Commission on Radiological Protection, 60, Japanese version. Japan Radioisotope Association, 1991 (in Japanese).

4. ICRP Publication 85: Avoidance of radiation injuries from medical interventional procedures, 85, Japanese version. Japan Radioisotope Association, 2003 (in Japanese).

5. Koenig TR, Wolff D, Mettler FA, Wagner LK. Skin injuries from fluoroscopically guided procedures: Part 1, characteristics of radiation injury. Am J Roentgenol 2001; 177: 3-11.

6. Ishikawa M, Soh I, Sueki H, Iijima M, Hayashi K, Wakugawa M. Two cases of chronic radiodermatitis caused by cardiac catheterization. The Nishinihon Journal of Dermatology 1999; 61: 731-736 (in Japanese). 
7. Soh I, Ishikawa M, Iijima M. Radiodermatitis following cardiac catheter and hepatic artery embolization. The Japanese Journal of Clinical Dermatology 2000; 54: 7-10 (in Japanese).

8. Soh I. Diagnosis and treatment of radiodermatitis following interventional radiological procedures. Jpn J Interv Cardiol 2002; 17: 357-360 (in Japanese).

9. The guidelines on prevention of radiation-related skin disorders following interventional radiological procedures-Q\&A and Discussions. Booklet Series 3. Japan Association on Radiological Protection in Medicine, 2004 (in Japanese).

10. Koga S. Comments on ICPR's draft recommendations by Japan Association on Radiological Protection in Medicine. Newsletter of Japan Association of Radiological Protection in Medicine 2000; 29: 73 -74 (in Japanese).

11. Saito I. Methods of description of parameters for pulsed fluoroscopy. Newsletter of Japanese Society of Circulatory Technology 2002; 5: 20-21 (in Japanese).

12. Awai $\mathrm{K}$, editor. Radiation exposure and protection in vascular imaging. Radiology and Medical Technology Library (17). Kyoto: Japanese Society of Radiological Technology, 1999; 22-25 (in Japanese)

13. Eguchi Y, Kinoshita J, Wakamatsu O, Ebihara Y, Kobayashi H, Uchiyama N. Clinical application of flat-panel detectors. Japanese Journal of Radiological Technology 2003; 59: 29-43 (in Japanese)

14. Kitai T, Ogawa T, Sano S. Patient absorbed dose in coronary angiography determined by the flat panel digital detector X-ray system. Japanese Journal of Radiological Technology 2003; 59: 423-426 (in Japanese).

15. Seguchi S, Ishikawa Y, Mizuno S, Saijou T, Nagao T, Nakamura A. Dose evaluation of a flat-panel detector system. Japanese Journal of Radiological Technology 2003; 59: 1438 -1443 (in Japanese).

16. Ichida T, Okusako K, Yokoyama K, Shougaki M, Ogawa T, Kawahata $\mathrm{H}$, et al. Clinical study with angiography system using a flat panel detecter. Japanese Journal of Radiological Technology 2004; 60: 1143 - 1152 (in Japanese).

17. Koenig TR, Mettler FA, Wagner LK. Skin injuries from fluoroscopically guided procedures: Part 2 , review of 73 cases and recommendations for minimizing dose delivered to patient. Am J Roentgenol 2001; 177: 13-20.

18. Mizutani H; Scientific Research Group, Japanese Society of Radiological Technology. Research on the measurement of patient dose and protection in IVR. Japanese Journal of Radiological Technology 2003; 59: 369-381 (in Japanese).

19. Awai K, Aoki Y, Ito T, Ohotakeno H, Fukutomi Y, Fujimoto N, et al. Study reports in radiation exposure for a medical workers and protective clothing in recent X-ray study. Japanese Journal of Radiological Technology 1998; 54: 687-696 (in Japanese).

20. Mizutani H, Kobayashi Y, Saida T, Saito I, Saito Y. A report of "an examination group of the Standard Radioprotective Implement for the Angiographic Operators". Japanese Journal of Radiological Technology 2001; 57: 1469-1478 (in Japanese).

21. Awai K, Oonobori K, Hayashida A, Fujii S, Mizutani H, Miyake H, et al. Report from the group of aerial-dosis measurement in case of IVR (PTCA). Japanese Journal of Radiological Technology 2001; 57: 33-48 (in Japanese).

22. Inoue S, Matsumoto M, Matsuzawa R. Examination of optimal radiation quality in the lead equivalent examination of X-ray protective clothing. Japanese Journal of Radiological Technology 2004; 60: $1682-1687$ (in Japanese)

23. Working session report: A report on radiation exposure due to damage to protective clothing and guidelines for the management of protective clothing. Japanese Journal of Radiological Technology 2000; 56: 552-557 (in Japanese).

24. Itou T. Evaluation and routine testing in medical imaging departments-Part 2-8: Constancy tests-protective shielding, -barriers and -devices JIS Z4752-2-8 (IEC 61223-2-8). Japanese Journal of Radiological Technology 2005; 61: 1104-1105 (in Japanese).

25. Ikuse J. IEC standards regarding the constancy tests and aceptance tests. Japanese Journal of Radiological Technology 2001; 57: 49-50 (in Japanese).

26. Iino T. An effective period of the use of medical appliances. Japanese Journal of Radiological Technology 2002; 58: 274-279 (in Japanese)

27. Shinohara F, Ito T, Shitara A, Kato Y. Evaluation and routine test- ing in medical imaging departments-Part 2-5: Contancy testsimaging display devices, JIS Z 4752-2-5: 2001 (Committee News). Japanese Journal of Radiological Technology 2000; 58: 631-633 (in Japanese).

28. Shinohara F, Abe M, Miyazaki S. Evaluation and routine testing in medical imaging departments-Part 3-3: Acceptance tests-imaging performance of X-ray equipment for digital subtraction angiography (DSA) JIS Z 4752-3-3: 2003. Japanese Journal of Radiological Technology 2003; 59: 621 -624 (in Japanese).

29. Fukunaga Y, Matsuoka N, Mae T, Kouno T. Trial manufacture of a plunger to reduce finger exposure. Kaku Igaku 2001; 38: 113-123 (in Japanese).

30. Inaba T, Nakayama K, Mizutani H. Radiology dermatitis due to thallium chloride. Practical Dermatology 2001; 23: 919-921 (in Japanese).

31. Fukuda H. Radiation-induced skin injuries. Kaku Igaku 2003; 40: 213-219 (in Japanese).

32. Nishitani H, Yasutomo M, Tominaga M, Fukui H, Yagi H. Radiation exposure in CT. Nippon Igaku Hoshasen Gakkai Zasshi 2002; 62: $347-351$ (in Japanese)

33. Nishizawa K, Matsumoto M, Iwai K, Maruyama T. Survey of CT practice in Japan and collective effective dose estimation. Nippon Igaku Hoshasen Gakkai Zasshi 2004; 64: 151 - 158 (in Japanese).

34. ICRP Publication 87: Managing patient dose in computed tomography, 87, Japanese version. Japan Radioisotope Association, 2004 (in Japanese).

35. Pharmaceutical and Food Safety Bureau, Ministry of Health, Labor, and Welfare. Effects of drugs and X-ray CT instruments on the function of an implantable pacemaker (Medtronic InSync 8040). Medical Device Safety Information No. 213, 2005 (in Japanese).

36. ICRP Publication 84: Pregnancy and medical radiation, 84, Japanese version. Japan Radioisotope Association, 2002 (in Japanese).

\section{Appendix}

Chair:

- Ryozo Nagai, Department of Cardiovascular Medicine, Graduate School of Medicine, The University of Tokyo

Members:

- Kazuo Awai, Department of Radiology, National Hospital Organization Fukui National Hospital

- Yasunobu Hirata, Department of Cardiovascular Medicine, Graduate School of Medicine, The University of Tokyo

- Yoshito Iesaka, Department of Internal Medicine, Tsuchiura Kyodo General Hospital

- Sugao Ishiwata, Department of Internal Medicine, Cardiovascular Center, Toranomon Hospital

- Tohru Kikuchi, Jichi Medical School Radioisotope Center

- Hiroshi Mizutani, Department of Radiology, Matsuyama Red Cross Hospital

- Hiromu Nishitani, Department of Radiology, The University of Tokushima

- Harumizu Sakurada, Tokyo Metropolitan Hiroo Hospital

- Morio Shoda, Department of Cardiology, The Heart Institute of Japan, Tokyo Women's Medical University

- Inketsu Soh, Department of Dermatology, Showa University Northern Yokohama Hospital

- Shigemasa Tani, Department of Cardiology, Surugadai Nihon University Hospital

- Ichiro Yamaguchi, Yamagata Prefecture, Murayama Public Health Center

- Hiroshi Yamashita, Department of Cardiovascular Medicine, Graduate School of Medicine, The University of Tokyo

Independent Assessment Committee:

- Tohru Izumi, Department of Cardio-angiology, Kitasato University, School of Medicine

- Katsuo Kanmatsuse, Tokyo Heart Center

- Tohru Ohe, Department of Cardiovascular Medicine, The Sakakibara Heart Institute of Okayama

- Tetsu Yamaguchi, Toranomon Hospital

(The affiliations of the members are as of March 2010) 